Sobre soluções periódicas de equações diferenciais com retardo e impulsos 


\title{
Sobre soluções periódicas de equações diferenciais com retardo e impulsos
}

\author{
André Luiz Furtado \\ Orientadora: Profa. Dra. Márcia Cristina Anderson Braz Federson \\ Coorientador: Prof. Dr. Pierluigi Benevieri
}

Tese apresentada ao Instituto de Ciências Matemáticas e de Computação - ICMC-USP, como parte dos requisitos para obtenção do título de Doutor em Ciências - Matemática . VERSÃO REVISADA

USP - São Carlos

Maio de 2012 
Ficha catalográfica elaborada pela Biblioteca Prof. Achille Bassi e Seção Técnica de Informática, ICMC/USP, com os dados fornecidos pelo(a) autor(a)

\begin{tabular}{|c|c|}
\hline \multirow[t]{3}{*}{ F992s } & $\begin{array}{l}\text { Furtado, André Luiz } \\
\text { Sobre soluções periódicas de equações diferenciais } \\
\text { com retardo e impulsos / André Luiz Furtado; } \\
\text { orientadora Márcia Cristina Anderson Braz Federson; } \\
\text { co-orientador Pierluigi Benevieri. -- São Carlos, } \\
2012 \text {. } \\
\quad 92 \text { p. }\end{array}$ \\
\hline & $\begin{array}{l}\text { Tese (Doutorado - Programa de Pós-Graduação em } \\
\text { Matemática) -- Instituto de Ciências Matemáticas e } \\
\text { de Computação, Universidade de São Paulo, } 2012 \text {. }\end{array}$ \\
\hline & $\begin{array}{l}\text { 1. equações diferenciais com retardamento. } 2 . \\
\text { equações impulsivas. I. Federson, Márcia Cristina } \\
\text { Anderson Braz, orient. II. Benevieri, Pierluigi, co- } \\
\text { orient. III. Título. }\end{array}$ \\
\hline
\end{tabular}


À minha mãe, Dalva de Oliveira. 



\section{Agradecimentos}

Agradeço especialmente à minha mãe, Dalva de Oliveira, pelo carinho, amor, educação e apoio que sempre me deu. Pelo exemplo de sensatez, força e equilíbrio. Obrigado por tudo!

Ao meu pai, Pedro Ivo Furtado (em memória), pela educação e por ter me convencido a fazer graduação em matemática.

À minha orientadora, Márcia Federson, por ter aceitado me orientar, pelo apoio e incentivo e pelo papel fundamental que cumpriu na minha formação como matemático.

Ao meu co-orientador, Pierluigi Benevieri, pela atenção, seriedade e exemplo de profissionalismo com que acompanhou e participou deste trabalho.

Agradeço a todos os professores que tive ao longo da minha vida. De maneira especial, a Hamilton Araújo Bicalho (em memória), professor de física em minha graduação e um inesquecível exemplo de retidão e seriedade profissional.

Aos meus colegas de doutorado e amigos, Cleber e Suzete, sou grato pela amizade, companhia e pela ajuda que, com excepcional boa vontade, deram sempre que precisei.

Por fim, agradeço à FAPESP pelo apoio financeiro. 



\section{Resumo}

Neste trabalho, apresentamos condições suficientes para a existência e a unicidade de soluções periódicas para equações diferenciais funcionais com retardo e impulsos. Os resultados sobre existência estão ancorados num Teorema de Continuação de Jean Mawhin. Por outro lado, as condições que garantem a unicidade de soluções periódicas são condições do tipo Lipschitz. 



\section{Abstract}

In this work, we present sufficient conditions for the existence and the uniqueness of periodic solutions for retarded functional differential equations with impulses. The results on the existence of periodic solutions are anchored by a Jean Mawhin continuation theorem. Moreover, the conditions that guarantee the uniqueness of the periodic solutions are Lipschitz type. 



\section{Sumário}

Introdução

1 Aplicação da teoria do grau às equações abstratas

2 Existência e unicidade de solução periódica para EDFR's impulsivas

2.1 Introdução . . . . . . . . . . . . . . . . . . . . 31

2.2 Existência de solução periódica . . . . . . . . . . . . . . . . 33

2.3 Unicidade de solução periódica . . . . . . . . . . . . . . . . . . 56

3 Existência e unicidade de solução periódica para EDFR's impulsivas neutras $\quad 61$

3.1 Introdução . . . . . . . . . . . . . . . . . . . . . 61

3.2 Uma equação não impulsiva auxiliar . . . . . . . . . . . . . . . . . 66

3.3 Existência de solução periódica . . . . . . . . . . . . . . . . . . . 69

3.4 Unicidade de solução periódica . . . . . . . . . . . . . . . . . . 85 



\section{Introdução}

\section{Apresentação}

O objetivo deste trabalho é investigar a questão da existência e unicidade de solução periódica para equações diferenciais funcionais com retardamento (escrevemos EDFRs) com ação impulsiva.

Diversos artigos publicados recentemente tratam da questão da existência ou da existência e unicidade de solução periódica para EDFRs em que o retardo é constante ou discreto. Ver, por exemplo, [15], [26], [27] e [32]. Nossa contribuição no Capítlo 2 consistiu em encontrar condições suficientes para a existência e unicidade de solução periódica para EDFRs com condições impulsivas não lineares e com retardo contínuo.

No Capítulo 3, apresentamos condições suficientes para a existência e unicidade de solução periódica para uma classe de equações diferenciais funcionais neutras com ação impulsiva. Da mesma forma que no segundo capítulo, no Capítulo 3 tratamos de equações com retardo contínuo e com condições impulsivas não lineares. Nesse capítulo, apresentamos um método para encontrar uma equação diferencial neutra sem impulsos, associada à equação impulsiva original, de tal modo que da existência e unicidade de solução periódica para tal equação não impulsiva podemos inferir o mesmo para a equação impulsiva. Até onde sabemos não existem trabalhos publicados em que se utiliza tal método para equações diferenciais neutras com ação impulsiva. Como sugestão de leitura de trabalhos recentes referentes ao problema da existência ou da existência e unicidade de solução periódica para equações diferencias neutras impulsivas 
sugerimos [11], [18], [19] e [30].

Os resultados que obtivemos sobre a existência de solução periódica, tanto no Capítulo 2 como no Capítulo 3, se apoiam num Teorema de Continuação de Mawhin que, por sua vez, tem a Teoria do Grau Topológico como pré-requisito. Por essa razão, julgamos por bem dedicar o primeiro capítulo deste trabalho a um resumo sobre tal teoria. Salientamos que a profundidade e abrangência de tal resumo se limitam ao necessário para o bom entendimento do Teorema de Continuação de Mawhin que utilizamos.

\section{Motivação}

Nos últimos anos, as equações diferencias funcionais com retardo e impulsos tem sido objeto de intensa pesquisa. Isto se deve a duas razões principais. Uma delas consiste na grande utilidade de tais equações na modelagem de diversos fenômenos que ocorrem nas mais diversas áreas do conhecimento como, por exemplo, dinâmica populacional, engenharia, física, química, medicina e economia. Além disso, o estudo de tais equações é fortemente motivado pelo interesse intrinsicamente matemático que possuem.

As equações diferenciais ordinárias e parciais estão consolidadas como eficazes modelos matemáticos de numerosos fenômenos reais. Tais equações são poderosas representações teóricas de processos de evolução em que a taxa de variação do estado do processo em cada instante $t$ depende do estado do processo nesse instante. No entanto, existe uma grande quantidade de fenômenos reais em que a taxa de variação do estado em cada instante depende, não somente do estado do processo nesse instante, mas também do histórico de estados do fenômeno. Dessa forma, para tais casos, as equações diferenciais ordinárias e parciais não se apresentam como os modelos mais apropriados e faz-se necessário a utilização de outras ferramentas teóricas que descrevam tais fenômenos de forma mais realista. Tais ferramentas são as equações diferencias funcionais com retardo. 
Por outro lado, muitos fenômenos reais são processos de evolução que se caracterizam por sofrer intensas e abruptas mudanças de estado. É comum referir-se a tais fenômenos como fenômenos impulsivos. Para modelar matematicamente os fenômenos impulsivos, de forma a incorporar suas bruscas alterações de estado, é usual considerar que o tempo de ocorrência dessas alterações seja desprezível em relação à duração do processo. Assim, é natural que nos modelos matemático de tais fenômenos, as mudanças abruptas de estado referidas acima sejam tratadas como instantâneas. Os instantes em que ocorrem tais alterações repentinas de estado são comumente chamados de momentos impulsivos e as ferramentas teóricas que possibilitam a modelagem matemática dos fenômenos impulsivos são as equações diferenciais impulsivas.

Podemos concluir, a partir do que foi exposto acima, que a modelagem matemática de fenômenos impulsivos em que a taxa de variação de estado em cada instante depende do estado atual e do histórico dos estados anteriores requer, como ferramentas teóricas, as equações diferencias funcionais com retardo bem como as equações diferenciais impulsivas. Surge assim, de forma natural, uma importante motivação para o estudo das equações diferencias funcionais com retardo e impulsos (EDFR's impulsivas). Além disso, como mencionamos acima, tanto as equações diferencias funcionais com retardo como as equações diferenciais impulsivas, apresentam diversas propriedades matemáticas distintas das equações diferenciais ordinárias e parciais, o que lhes proporciona um marcado interesse puramente matemático.

A título de exemplos para a última afirmação, vale mencionar que no contexto das equações diferenciais funcionais com retardo um problema de valor inicial tem como dado inicial, não um ponto do espaço euclidiano, como ocorre com tais problemas no caso das equações diferenciais ordinárias e parciais, mas sim uma função. Como era de se esperar, fatos como esse acarretam em várias diferenças entre a teoria das equações diferencias com retardo e as equações diferenciais ordinárias e parciais. Sugerimos como leitura sobre as equações diferenciais com retardo as referências [1], [2], [16], [21], [22], [23] e [34]. 
Com relação às particularidades matemáticas das equações diferenciais impulsivas, mencionamos, como exemplo, o fato de que, ao contrário do que ocorre no caso das equações diferenciais ordinárias e parciais, as soluções de tais equações nem sequer são contínuas nos pontos correspondentes aos impulsos e, portanto, não satisfazem em todos os pontos a igualdade que expressa a equação. Isso acarreta em alterações das condições para que algumas propriedades bem conhecidas sejam satisfeitas e até mesmo na definição de alguns conceitos. Citamos, como exemplo, a depenência contínua com relação aos dados iniciais e propriedades qualitativas como estabilidade. Como recomendação para leitura sobre a teoria das equações diferenciais impulsivas, citamos [4], [5], [6], [7], [33], [24]

Recentemente, numerosos artigos dedicados às EDFR's impulsivas tem sido publicados em diversas revistas científicas internacionais. Aplicações, existência e unicidade de soluções, comportamento assintótico, estabilidade e limitação, são alguns exemplos da ampla gama de temas abordados em tais publicações. No presente trabalho, nos dedicamos a investigar a questão da existência e unicidade de solução periódica para algumas classes de EDFR's impulsivas.

Antes de apresentarmos formalmente as EDFR's impulsivas, exporemos alguns casos concretos de situações reais que devem ser modeladas por equações diferencias funcionais com retardo, por equações diferenciais impulsivas e, finalmente, daremos um exemplo de situação real modelada EDFR's impulsivas.

Iniciamos com um exemplo da vida cotidiana que julgamos de grande valor didático e que pode ser encontrado em [22]. Imaginemos a situação corriqueira que consiste em um indivíduo regulando a temperatura da água do seu banho girando a torneira do chuveiro. Denote por $T_{d}$ a temperatura que o indivíduo deseja que a água tenha e por $T(t)$ a temperatura da água do chuveiro no instante $t$. Desejamos encontrar a equação que descreve a variação da temperatura da água do chuveiro em função da diferença entre a temperatura da água percebida pelo indivíduo e a temperatura desejada $T_{d}$. 
Note que a temperatura da água percebida pelo indivíduo no instante $t$ é a temperatura da água no chuveiro no instante $t-\tau$, sendo $\tau$ o intervalo de tempo necessário para que a água que sai do chuveiro chegue ao indivíduo. Dessa forma, é de se esperar que a equação que buscamos seja uma equação com retardo, pois a variação da temperatura da água do chuveiro em cada instante $t$ será dada em função da sua temperatura num instante anterior $t-\tau$.

Denotemos por $\alpha(t)$ o ângulo de rotação da torneira do chuveiro no instante $t$. É natural supor que a taxa de variação de $\alpha$, isto é, $\alpha^{\prime}(t)$, seja proporcional à diferença entre a temperatura da água que chega ao indivíduo e a temperatura $T_{d}$. Assim, denotando por $\kappa$ a constante de proporcionalidade, obtemos a equação

$$
\alpha^{\prime}(t)=\kappa\left[T(t-\tau)-T_{d}\right]
$$

Como, por outro lado, a taxa de variação da temperatura da água no chuveiro é proporcional à taxa de variação do ângulo de rotação da torneira, com constante de proporcionalidade, digamos $\imath$, então, utilizando a equação acima, podemos concluir que a dinâmica da variação da temperatura da água no chuveiro é descrita pela equação diferencial com retardo discreto

$$
T^{\prime}(t)=\kappa \imath^{-1}\left[T(t-\tau)-T_{d}\right]
$$

A seguir, apresentamos um caso de dinâmica populacional modelado por uma equação diferencial com retardo contínuo. Suponha que uma determinada espécie animal esteja sujeita à infestação parasitária de tal maneira que, no início da infestação, o sistema imunológico do hospedeiro contra o parasita seja baixo, permitindo a rápida proliferação do mesmo no organismo do hospedeiro. Suponha, ainda, que à medida que a população parasitária cresce, o sistema imunológico do hospedeiro se fortalece a ponto de ocasionar a diminuição na taxa de crescimento populacional parasitária e a subsequente diminuição de tal população. 
Note que, em cada instante, a variação da população parasitária dependerá não somente da população nesse instante, mas, também, do histórico da variação populacional. De fato, se num determinado momento do início da infestação a população de parasitas for $N$, então, como o sistema imunológico do hospedeiro ainda é baixo, a população parasitária continuará aumentando. No entanto, como o aumento do número de parasitas provoca o aumento da eficiência do sistema imunológico do hospedeiro, então, nessa fase, com o mesmo valor $N$ de indivíduos parasitários, ocorrerá uma diminuição subsequente de sua população.

Dessa forma, podemos concluir que a dinâmica populacional dos parasitas será descrita por uma equação diferencial com retardo. De acordo com [23], tal equação é a seguinte equação diferencial com retardo contínuo

$$
N^{\prime}(t)=r N\left[1-\frac{N(t)}{K}-\int_{0}^{t} N(s) G(t-s) d s\right]
$$

sendo $N(t)$ a população parasiária no instante $t, G$ uma função chamada de função memória e $r$ e $K$ constantes dadas.

Outro exemplo de aplicação das equações diferencias funcionais com retardo que, pela sua importância, julgamos pertinente apresentar, se refere à modelagem matemática dos efeitos do uso de drogas quimioterápicas no ciclo celular de células cancerosas. Sem a pretensão de sermos rigorosos na descrição dos processos biológicos envolvidos, podemos dizer que a droga absorvida por uma célula cancerosa num instante $t$ irá influenciar seu ciclo celular após um intervalo de tempo $h$. E mais, o lapso de tempo entre a absorção da droga por parte da célula e os efeitos da mesma no ciclo celular, em geral, não é o mesmo para as três fases do ciclo celular (intérfase, fase mitótica e mitose). Denotando por $h_{i}$ tais intervalos de tempo e por $x_{i}(t)$, $i=1,2,3$, a quantidade de células cancerosas em cada instante $t$ e em cada umas das três fases do ciclo celular, obteve-se, como modelo da dinâmica celular cancerosa, o seguinte sistema de 
equações diferencias funcionais com retardo discreto ([22]):

$$
\left\{\begin{array}{l}
x_{1}^{\prime}(t)=\alpha\left[a_{3}+u_{3}\left(t-h_{3}\right)\right] x_{3}\left(t-h_{3}\right)-\left[a_{1}+u_{1}\left(t-h_{1}\right)\right] x_{1}\left(t-h_{1}\right) \\
x_{2}^{\prime}(t)=\left[a_{1}+u_{1}\left(t-h_{1}\right)\right] x_{1}\left(t-h_{1}\right)-\left[a_{2}+u_{2}\left(t-h_{2}\right)\right] x_{2}\left(t-h_{2}\right) \\
x_{3}^{\prime}(t)=\left[a_{2}+u_{2}\left(t-h_{2}\right)\right] x_{2}\left(t-h_{2}\right)-\left[a_{3}+u_{3}\left(t-h_{3}\right)\right] x_{3}\left(t-h_{3}\right) .
\end{array}\right.
$$

Aqui, $\alpha$ denota a taxa de reprodução celular, $u_{i}(t)$ denota a quantidade de droga em atuação na célula no instante $t$ e na fase $i$ e a função $t \mapsto a_{i}+u_{i}(t)$ descreve a quantidade de células mudando para a fase $i$ em cada instante $t$.

Como sugerem os exemplos acima, as equações diferencias funcionais com retardo se aplicam às mais diversas áreas do conhecimento. Nas referências [22] e [23] há uma grande quantidade de exemplos de situações reais modeladas por equações com retardo discreto, como (1) e (3) e por retardo contínuo, como (2). Segundo [22], a grande maioria das situações reais modeladas por EDFR's o são por equações com retardo discreto, que apresentam a forma geral

$$
x^{\prime}(t)=g\left(t, x\left(t-h_{1}(t)\right), \ldots, x\left(t-h_{k}(t)\right)\right), \quad h_{i}(t) \geq 0
$$

ou por equações com retardo contínuo que apresentam a forma geral

$$
\left.x^{\prime}(t)=g\left(t, \int_{t-h(t)}^{t} K(t, s, x(s)) d s\right)\right), \quad h(t) \geq 0
$$

conhecidas como equações integro-diferenciais do tipo Volterra.

As equações diferencias funcionais com retardo que estudamos no Capítulo 2 apresentam a seguinte forma geral

$$
x^{\prime}(t)=f\left(t, x_{t}\right)
$$

Dados dois números reais $r>0$ e $t \geq 0$ e uma função $x:[-r,+\infty) \longrightarrow \mathbb{R}$, denotamos por $x_{t}$ a 
função real dada por $x_{t}(\theta)=x(t+\theta), \theta \in[-r, 0]$. Note que, a grosso modo, a função $x_{t}$ retrata a restrição da função $x$ a uma $r$-vizinhaça à esquerda do ponto $t$. Dessa forma, ao interpretarmos o número $t$ como o instante $t$, podemos interpretar a $r$-vizinhaça à esquerda de $t$ como o passado em relação ao instante $t$. Isso nos permite concluir que a função $x_{t}$ assume um importante papel na teoria das equações diferencias funcionais com retardo.

De fato, tanto a equação (4) como a equação (5) são casos particulares de (6). Com efeito, obtemos (4) a partir de (6), definindo

$$
f(t, \varphi):=g\left(t, \varphi\left(-h_{1}(t)\right), \ldots, \varphi\left(-h_{k}(t)\right)\right)
$$

Por outro lado, para obter (5) a partir de (6), basta definir

$$
f(t, \varphi):=g\left(t, \int_{-h(t)}^{0} K(t, t+s, \varphi(t+s)) d s\right)
$$

Dessa forma, vemos que a equação (6) apresenta um razoável grau de generalidade.

Como mencionamos anteriormente, muitos fenômenos reais possuem a característica de, em curtos intervalos de tempo, sofrer abrupta mudança de estado. As equações diferenciais impulsivas se apresentam como eficientes modelos para tais fenômenos quando tais intervalos de tempo podem ser considerados desprezíveis em relação à duração de todo o processo. Por exemplo, considere o caso de um tratamento médico em que o paciente deve ingerir um determinado medicamento nos instantes $t_{1}, t_{2}, \ldots, t_{p}$. Denotemos por $\delta_{i}$ a quantidade de medicamento ingeriada no instante $t_{i}, i=1, \ldots, p$, por $x(t)$ a quantidade de medicamento no sistema digestivo do paciente e por $y(t)$ a quantidade de medicamento no seu sistema circulatório, ambos no instante $t$. De acordo com [24], a variação da quantidade de medicamento no organismo do 
paciente durante o tratamento é dado pelo seguinte sistema de equações diferenciais impulsivas

$$
\begin{cases}x^{\prime}(t)=-k_{1} x(t), \quad y^{\prime}(t)=k_{2} y(t)+k_{1} x(t), & t \in[0, \tau] t \neq t_{1}, \ldots, t_{p} \\ x\left(t_{i}^{+}\right)=x\left(t_{i}^{-}\right)+\delta_{i} \quad y\left(t_{i}^{+}\right)=y\left(t_{i}^{-}\right), & i=1 \ldots, p .\end{cases}
$$

Aqui, $k_{1}$ e $k_{2}$ são constantes dadas, $\tau$ é o instante a partir do qual se pode considerar finalizado o tratamento e $x\left(t_{i}^{+}\right), x\left(t_{i}^{-}\right), y\left(t_{i}^{+}\right), y\left(t_{i}^{-}\right)$denotam os limites laterais de $x$ e $y$ em $t_{i}$.

Vejamos um processo ecológico que pode ser modelado por equações diferenciais com retardo e impulsos. Sabe-se que duas espécies de plânctons que coexistem no mesmo ambiente e se nutrem dos mesmos nutrientes comportam-se como espécies em competição. De fato, cada espécie produz substâncias químicas tóxicas à espécie concorrente. A quantidade dessas substâncias depende do tamanho da população da outra espécie. Além disso, note que a produção de tais substâncias em cada instante $t$ depende da população da outra espécie no instante $t-\tau$, sendo $\tau$ o tempo necessário para a produção das substâncias tóxicas. Assim, podemos concluir que as equações diferencias com retardo devem desempenhar um importante papel em um modelo matemático realista da dinâmica populacional de mais de uma espécie de plânctons que compartilham o mesmo ambiente.

Além disso, os plânctons possuem a característica de em determinadas situações apresentarem um repentino crescimento populacional, conhecido como "florescimento" ou bloom, e que são ocasionados por diversos fatores, por exemplo, poluição e clima favorável. Em [20], os autores modelaram tais explosões populacionais como efeitos impulsivos na dinâmica populacional dos plânctons estudados. Mais precisamente, os autores encontraram o seguinte sistema de equações diferenciais com retardo e impulsos, para modelar a dinâmica populacional de duas 
espécies de plânctons em um mesmo ambiente e sob o efeito de florescimento:

$$
\begin{cases}N_{1}^{\prime}(t)=N_{1}\left[k_{1}(t)-\alpha_{1}(t) N_{1}(t)-\beta_{12}(t) N_{2}(t)-\gamma_{1}(t) N_{1}(t) N_{2}(t-\tau(t))\right], & t \geq 0, t \neq t_{k} \\ N_{2}^{\prime}(t)=N_{2}\left[k_{2}(t)-\alpha_{2}(t) N_{2}(t)-\beta_{21}(t) N_{1}(t)-\gamma_{2}(t) N_{1}(t-\tau(t)) N_{2}(t)\right], \quad t \geq 0, t \neq t_{k} \\ N_{1}\left(t_{k}^{+}\right)-N_{1}\left(t_{k}\right)=b_{1 k} N_{1}\left(t_{k}\right), \quad N_{1}\left(t_{k}^{+}\right)-N_{1}\left(t_{k}\right)=b_{1 k} N_{1}\left(t_{k}\right)\end{cases}
$$

Ao leitor interessado em encontrar aplicações das equações diferenciais funcionais com retardo e impulsos, recomendamos os artigos [3], [15], [25], [28], [29], [32], [35] e [36]. 


\section{Aplicação da teoria do grau às equações abstratas}

Este capítulo se destina a uma breve e resumida apresentação da teoria do grau topológico, ferramenta fundamental para a obtenção dos resultados referentes à existência de soluções periódicas para os problemas que estudamos nos dois próximos capítulos.

Sejam $\Omega \subset \mathbb{R}^{n}$ aberto limitado e $C^{k}\left(\bar{\Omega}, \mathbb{R}^{n}\right), k \in \mathbb{N}$, o espaço das funções $\varphi: \Omega \longrightarrow \mathbb{R}^{n}$ que são $k$ vezes diferenciáveis em $\Omega$ e tais que elas e suas derivadas até ordem $k$ podem ser extendidas continuamente a $\bar{\Omega}$. Consideramos em $C^{k}\left(\bar{\Omega}, \mathbb{R}^{n}\right)$ a norma dada por

$$
\|\varphi\|_{C^{k}}=\max _{0 \leq j \leq k} \sup _{t \in \Omega}\left\|\varphi^{(j)}(t)\right\|
$$

Sejam $\varphi \in C^{1}\left(\bar{\Omega}, \mathbb{R}^{n}\right)$ e $S=\left\{t \in \Omega ; J_{\varphi}(t)=0\right\}$, sendo $J_{\varphi}(t)$ o determinante jacobiano de $\varphi$ em $t$. Os elementos de $S$ serão chamados de pontos críticos de $\varphi$ e os pontos $b \in \mathbb{R}^{n}$ que não são imagem de pontos críticos de $\varphi$ serão chamados de valores regulares de $\varphi$. Além disso, no que se segue, utilizaremos a notação $\partial \Omega$ para representar a fronteira do conjunto $\Omega$.

Sejam $\varphi \in C^{1}\left(\bar{\Omega}, \mathbb{R}^{n}\right)$ e $b \notin \varphi(\partial \Omega)$ um valor regular de $\varphi$. O grau topológico de $\varphi$ em relação a $\Omega$ no ponto $b$ é um número inteiro que, na sequência, definiremos de maneira precisa.

O próximo resultado será crucial para que possamos definir o grau topológico $\operatorname{deg}(\varphi, \Omega, b)$ no caso em que $\Omega \subset \mathbb{R}^{n}$ for aberto limitado, $\varphi \in C^{1}\left(\bar{\Omega}, \mathbb{R}^{n}\right)$ e $b \notin \varphi(\partial \Omega)$ for um valor regular 
de $\varphi$.

Proposição 1.1. Se $\varphi \in C^{1}\left(\bar{\Omega}, \mathbb{R}^{n}\right)$ e $b \notin \varphi(S) \cup \varphi(\partial(\Omega))$, então $\varphi^{-1}(\{b\})$ será finito.

Demonstração. Sejam $\varphi \in C^{1}\left(\bar{\Omega}, \mathbb{R}^{n}\right)$ e $b \notin \varphi(S) \cup \varphi(\partial(\Omega))$. Se $\varphi^{-1}(\{b\})=\emptyset$ o resultado é trivial. Suponha que $\varphi^{-1}(\{b\}) \neq \emptyset$. Como $b \notin \varphi(S)$, então $J_{\varphi}(t) \neq 0$ para cada $t \in \varphi^{-1}(\{b\})$. Então, pelo Teorema da Função Inversa, para cada $t \in \varphi^{-1}(\{b\})$, existe uma bola aberta $B(t) \subset$ $\Omega$, de centro em $t$, tal que a restrição de $\varphi$ a $B(t)$ é um difeomorfismo. Assim, podemos escrever

$$
\varphi^{-1}(\{b\}) \subset \bigcup_{t \in \varphi^{-1}(\{b\})} B(t)
$$

e considerar que as bolas $B(t), t \in \varphi^{-1}(\{b\})$, são duas a duas disjuntas.

Por outro lado, como $\varphi$ é contínua, então $\varphi^{-1}(\{b\})$ é fechado. Além disso, $\varphi^{-1}(\{b\})$ está contido em $\Omega$, que é limitado. Assim, concluímos que $\varphi^{-1}(\{b\})$ é compacto. Dessa forma, pelo Teorema de Borel-Lebesgue, é possível extrair de $\{B(t)\}_{t \in \varphi^{-1}(\{b\})}$ uma subcobertura finita

$$
\varphi^{-1}(\{b\}) \subset\left\{B\left(t_{1}\right), \ldots, B\left(t_{j}\right)\right\}
$$

Daí, como $\varphi$ é injetiva em cada bola $B\left(t_{i}\right)$ e $\varphi\left(t_{i}\right)=b$, obtemos

$$
\varphi^{-1}(\{b\})=\left\{t_{1}, \ldots, t_{j}\right\}
$$

finalizando a demonstração.

Neste momento, estamos em condições de definir o grau topológico de Brower para ternas $\varphi, \Omega, b$ que satisfazem as condições enunciadas na Proposição 1.1.

Definição 1.2. O grau topológico de Brower de $\varphi \in C^{1}\left(\bar{\Omega}, \mathbb{R}^{n}\right)$ em relação a $\Omega$ num ponto 
$b \notin \varphi(S) \cup \varphi(\partial(\Omega))$ é o número inteiro

$$
\operatorname{deg}(\varphi, \Omega, b)=\left\{\begin{array}{cl}
\sum_{x \in \varphi^{-1}(\{b\})} \operatorname{sgn}\left(J_{\varphi}(x)\right), & \text { se } \varphi^{-1}(b) \neq \emptyset \\
0, & \text { se } \varphi^{-1}(b)=\emptyset
\end{array}\right.
$$

sendo $\operatorname{sgn}(a)=1$ se $a>0$ e $\operatorname{sgn}(a)=-1$ se $a<0$.

Exemplo 1.3. Vamos encontrar deg $(\varphi, \Omega, b)$, sendo $\Omega=(0,7 \pi / 2), \varphi: \Omega \longrightarrow \mathbb{R}, \varphi(t)=\cos t$ e $b=\pi / 8$. Primeiramente, para garantir que deg $(\varphi, \Omega, b)$ está bem definido, devemos verificar que $b \notin \varphi(\partial \Omega) \cup \varphi(S)$. Note que, $\partial \Omega=\{0,7 \pi / 2\}, S=\{t \in(0,7 \pi / 2)$; sen $t=0\}=$ $\{\pi, 2 \pi, 3 \pi\}, \varphi(\partial \Omega)=\{1,0\}, \varphi(S)=\{-1,1\}$ e, portanto, $\pi / 8 \notin \varphi(\partial \Omega) \cup \varphi(S)$. Encontremos $\operatorname{deg}(\varphi, \Omega, b)$. Denote $\left\{t_{1}, t_{2}, t_{3}\right\}=\varphi^{-1}(\{\pi / 8\})$. Então, por definição,

$$
\begin{aligned}
\operatorname{deg}(\cos ,(0,7 \pi / 2), \pi / 8) & =\operatorname{sgn}\left(-\operatorname{sen} t_{1}\right)+\operatorname{sgn}\left(-\operatorname{sen} t_{2}\right)+\operatorname{sgn}\left(-\operatorname{sen} t_{3}\right) \\
& =-1+1-1=-1
\end{aligned}
$$

A seguir, enunciamos uma das principais propriedades do grau. Tal proriedade desempenha importante papel nos resultados de existência de solução periódica que apresentaremos nos dois próximos capítulos. Ao leitor que deseje verificar as demonstrações de tal propriedade recomendamos a leitura dos trabalhos [8], [9], [10] ou [12].

Lema 1.4. Sejam $\Omega$ aberto limitado de $\mathbb{R}^{n}$ e $\operatorname{Id}: \bar{\Omega} \longrightarrow \mathbb{R}^{n}, \operatorname{Id}(x)=x$. Então

$$
\operatorname{deg}(I d, \Omega, b)= \begin{cases}1, & \text { se } b \in \Omega \\ 0, & \text { se } b \notin \Omega .\end{cases}
$$

Uma vez definido o grau topológico para a terna $\varphi, \Omega, b$, sendo $\varphi$ uma função em $C^{1}\left(\bar{\Omega}, \mathbb{R}^{n}\right)$, $\Omega \subset \mathbb{R}^{n}$ aberto limitado e $b$ valor regular de $\varphi$, nosso objetivo passa a ser, a partir de agora, apresentar a definição do grau topológico para um caso mais geral. A saber, desejamos definir o grau 
topológico $\operatorname{deg}(\varphi, \Omega, b)$ quando $b$ não for necesariamente um valor regular de $\varphi$ e tal função pertencer a uma classe de funções definidas em abertos limitados $\Omega$ de espaços de Banach $X$ e que não necessariamente pertencem a $C^{k}(\bar{\Omega}, X)$ se $k>0$. Na sequência, apresentaremos uma série de lemas que nos conduzirão de forma natural a tal definição.

O primeiro desses lemas afirma que o grau topológico de Brower é localmente constante na topologia de $C^{1}\left(\bar{\Omega}, \mathbb{R}^{n}\right)$.

Lema 1.5. Sejam $\varphi \in C^{1}\left(\bar{\Omega}, \mathbb{R}^{n}\right)$ e $b \notin \varphi(\partial \Omega) \cup \varphi(S)$. Então, existe uma vizinhança $U \subset$ $C^{1}\left(\bar{\Omega}, \mathbb{R}^{n}\right)$ de $\varphi$ tal que, para cada $\psi \in U$, vale:

(i) $b \notin \psi(\partial \Omega)$;

(ii) $\operatorname{Se} \psi(t)=b$, então $J_{\psi}(t) \neq 0$;

(iii) $\operatorname{deg}(\psi, \Omega, b)=\operatorname{deg}(\varphi, \Omega, b)$.

Demonstração. A demonstração do lema pode ser encontrada em [8] ou [9].

O resultado a seguir nos diz que o grau de Brower é constante com relação a $b$ em cada componente conexa de $\mathbb{R}^{n} \backslash(\varphi(\partial \Omega) \cup \varphi(S))$.

Lema 1.6. Sejam $\varphi \in C^{1}\left(\bar{\Omega}, \mathbb{R}^{n}\right)$ e $b, c$ dois pontos pertencentes à mesma componente conexa de $\mathbb{R}^{n} \backslash(\varphi(\partial \Omega) \cup \varphi(S))$, então

$$
\operatorname{deg}(\varphi, \Omega, b)=\operatorname{deg}(\varphi, \Omega, c)
$$

Demonstração. A demonstração deste lema pode ser encontrada em [8], [9] ou [12].

Com o próximo resultado vemos que se $\varphi \in C^{2}\left(\bar{\Omega}, \mathbb{R}^{\prime}\right)$, então o grau de Brower será constante com relação a $b$ em cada componente conexa de $\mathbb{R}^{n} \backslash \varphi(\partial \Omega)$. 
Lema 1.7. Sejam $\varphi \in C^{2}\left(\bar{\Omega}, \mathbb{R}^{n}\right)$. Se $b, c \notin \varphi(\partial \Omega) \cup \varphi(S)$ e estão na mesma componente conexa de $\mathbb{R}^{n} \backslash \varphi(\partial \Omega)$, então

$$
\operatorname{deg}(\varphi, \Omega, b)=\operatorname{deg}(\varphi, \Omega, c)
$$

Demonstração. Veja [8] ou [9].

Seja $C_{b}$ a componente conexa de $\mathbb{R}^{n} \backslash \varphi(\partial \Omega)$ que contém $b$. Como $\mathbb{R}^{n} \backslash \varphi(\partial \Omega)$ é aberto, segue-se que $C_{b}$ também é. Por outro lado, pelo Teorema de Sard, $\varphi(S)$ tem medida nula em $\mathbb{R}^{n}$. Dessa forma, podemos concluir que $\varphi(S)$ não contém $C_{b}$, isto é, $C_{b} \backslash \varphi(S) \neq \emptyset$. Este fato, juntamente com o Lema 1.7, nos permite definir o grau topológico $\operatorname{deg}(\varphi, \Omega, b)$, quando $\varphi$ pertencer a $C^{2}\left(\bar{\Omega}, \mathbb{R}^{n}\right)$ e $b$ for imagem de ponto singular de $\varphi$.

Definição 1.8. Sejam $\varphi \in C^{2}\left(\bar{\Omega}, \mathbb{R}^{n}\right)$ e $b \in \varphi(S)$ tal que $b \notin \varphi(\partial \Omega)$. Considere $C_{b}$ a componente conexa de $\mathbb{R}^{n} \backslash \varphi(\partial \Omega)$ que contém $b$. Definimos

$$
\operatorname{deg}(\varphi, \Omega, b)=\operatorname{deg}(\varphi, \Omega, c), \quad c \in C_{b} \backslash \varphi(S)
$$

sendo o membro direito da igualdade acima dado pela Definição 1.2.

Observe que, diferentemente da Definição 1.2, a Definição 1.8 estabelece o grau topológico em pontos não regulares. Por outro lado, houve uma maior exigência quanto à regularidade da função $\varphi$. De fato, enquanto na Definição 1.2 bastava que $\varphi$ pertencesse a $C^{1}(\bar{\Omega}, \mathbb{R})$, na Definição 1.8 exige-se que $\varphi$ pertença a $C^{2}(\bar{\Omega}, \mathbb{R})$.

Todavia, veremos, com os com os três próximos resultados, que podemos obter uma efetiva generalização das Definições 1.2 e 1.8. Mais precisamente, veremos que é possível definir o grau topológico $\operatorname{deg}(\varphi, \Omega, b)$ de funções $\varphi \in C\left(\bar{\Omega}, \mathbb{R}^{n}\right)$ em qualquer ponto $b \notin \varphi(\partial \Omega)$.

O primeiro desses resultados implica que o grau estabelecido na Definição 1.8 é localmente constante na topologia de $C^{1}(\Omega, \mathbb{R})$. 
Lema 1.9. Se $\varphi \in C^{2}\left(\bar{\Omega}, \mathbb{R}^{n}\right)$ e $b \notin \varphi(\partial \Omega)$, então existe uma vizinhança $U$ de $\varphi$, na topologia de $C^{1}\left(\bar{\Omega}, \mathbb{R}^{n}\right)$, tal que para cada $\psi \in U$, vale

(i) $b \notin \psi(\partial \Omega)$;

(ii) $\operatorname{deg}(\psi, \Omega, b)=\operatorname{deg}(\varphi, \Omega, b)$.

Demonstração. (Ideia) Se $b \notin \varphi(S)$ o resultado segue imediatamente do Lema 1.5. Se, por outro lado, $b \in \varphi(S)$, então o resultado segue do Teorema de Sard, da Definição 1.8 e do Lema 1.5. Para conferir os detalhes da demonstração, sugerimos ao leitor consultar [8] ou [9].

O resultado abaixo é conhecido como a propriedade da invariância por homotopia do grau topológico.

Lema 1.10. Se $H \in C^{2}\left(\bar{\Omega} \times[0,1], \mathbb{R}^{n}\right)$ e $b \notin H(\partial(\Omega) \times[0,1])$, então

$$
\operatorname{deg}\left(H\left(\cdot, t_{1}\right), \Omega, b\right)=\operatorname{deg}\left(H\left(\cdot, t_{2}\right), \Omega, b\right)
$$

quaisquer que sejam $t_{1}, t_{2} \in[0,1]$ fixados.

Demonstração. Sejam $H \in C^{2}\left(\bar{\Omega} \times[0,1], \mathbb{R}^{n}\right)$ e $b \notin H(\partial(\Omega) \times[0,1])$. Como as funcções $H$ e $\partial H / \partial x$ são uniformemente contínuas em $\bar{\Omega} \times[0,1]$, então, fixando $\tau \in[0,1]$, podemos concluir que para cada $\varepsilon>0$, existirá $\delta>0$ tal que

$$
t \in[0,1],|t-\tau|<\delta \Longrightarrow\|H(\cdot, t)-H(\cdot, \tau)\|_{C^{1}}<\varepsilon
$$

Disto, e do Lema 1.9, concluímos que se $\delta$ for suficientemente pequeno, então

$$
\operatorname{deg}(H(\cdot, t), \Omega, b)=\operatorname{deg}(H(\cdot, \tau), \Omega, b), \quad t \in(\tau-\delta, \tau+\delta)
$$


Dessa forma, mostramos que a função $d: t \mapsto \operatorname{deg}(H(\cdot, t), \Omega, b)$ é localmente constante em $[0,1]$. Daí, como $[0,1]$ é compacto e conexo, concluímos que $d$ é constante e o lema está provado.

A partir do próximo resultado, poderemos definir o grau de funções contínuas em $\bar{\Omega}$.

Lema 1.11. Se $\varphi \in C\left(\bar{\Omega}, \mathbb{R}^{n}\right)$ e $b \notin \varphi(\partial \Omega)$, então existirá uma vizinhança $U$ de $\varphi$, na topologia de $C^{1}\left(\bar{\Omega}, \mathbb{R}^{n}\right)$, tal que

$$
\operatorname{deg}\left(\psi_{1}, \Omega, b\right)=\operatorname{deg}\left(\psi_{2}, \Omega, b\right)
$$

quaisquer que sejam $\psi_{1}, \psi_{2} \in U$.

Demonstração. Sejam $\varphi \in C\left(\bar{\Omega}, \mathbb{R}^{n}\right)$ e $b \notin \varphi(\partial \Omega)$. Como $b \notin \varphi(\partial \Omega)$, a distância de $b$ a $\varphi(\partial \Omega)$ é positiva. Denotemos tal distância por $r$. Como, pelo Teorema de Aproximação de Weirstrass, $C^{2}\left(\bar{\Omega}, \mathbb{R}^{n}\right)$ é denso em $C\left(\bar{\Omega}, \mathbb{R}^{n}\right)$, então

$$
U=\left\{\psi \in C^{2}\left(\bar{\Omega}, \mathbb{R}^{n}\right) ;\|\varphi-\psi\|_{\infty}<r / 2\right\} \neq \emptyset
$$

Fixe $\psi_{1}, \psi_{2} \in U$ e defina $H: \bar{\Omega} \times[0,1] \longrightarrow \mathbb{R}, H(x, t)=t \psi_{1}(x)+(1-t) \psi_{2}(x)$. Afirmamos que $b \notin H(\partial \Omega \times[0,1])$. De fato, suponha, por absurdo, que exista $(x, t) \in \partial \Omega \times[0,1]$ tal que $H(x, t)=b$. Neste caso, como a distância entre $b$ e $\varphi(\partial \Omega)$ é $r$, então

$$
\|H(x, t)-\varphi(x)\|=\|b-\varphi(x)\| \geq r .
$$

Por outro lado, utilizando a expressão que define $H$, obtemos

$$
\begin{aligned}
\|H(x, t)-\varphi(x)\| & =\left\|t \psi_{1}(x)+(1-t) \psi_{2}(x)-t \varphi(x)-(1-t) \varphi(x)\right\| \\
& \leq t\left\|\psi_{1}(x)-\varphi(x)\right\|+(1-t)\left\|\psi_{2}(x)-\varphi(x)\right\| .
\end{aligned}
$$


Portanto,

$$
\|H(x, t)-\varphi(x)\| \leq t\left\|\psi_{1}-\varphi\right\|_{\infty}+(1-t)\left\|\psi_{2}-\varphi\right\|_{\infty}
$$

Daí, como $\psi_{1}, \psi_{2} \in U$, temos

$$
\|H(x, t)-\varphi(x)\|<t \frac{r}{2}+(1-t) \frac{r}{2}=\frac{r}{2}
$$

Como tal desigualdade está em contradição com (1.3) podemos concluir que $b \notin H(\partial \Omega \times[0,1])$. Como, além disso, $H \in C^{2}\left(\bar{\Omega}, \mathbb{R}^{n}\right)$, então, pelo Lema 1.10 , temos

$$
\operatorname{deg}((H(\cdot, 1), \Omega, b)=\operatorname{deg}((H(\cdot, 0), \Omega, b),
$$

isto é,

$$
\operatorname{deg}\left(\psi_{1}, \Omega, b\right)=\operatorname{deg}\left(\psi_{2}, \Omega, b\right)
$$

e o resultado está demonstrado.

Definição 1.12. Sejam $\varphi \in C(\bar{\Omega}) e b \notin \varphi(\partial \Omega)$. Definimos o grau toplógico de Brower de $\varphi$ em relação a $\Omega$ no ponto $b$, como sendo

$$
\operatorname{deg}(\varphi, \Omega, b)=\operatorname{deg}(\psi, \Omega, b), \quad \psi \in U
$$

sendo $U$ a vizinhança de $\varphi$, na topologia de $C^{1}\left(\bar{\Omega}, \mathbb{R}^{n}\right)$, cuja existência é garantida pelo Lema 1.11 .

Na sequência, estenderemos o conceito de grau topológico para uma certa classe de funções contínuas em abertos limitados de espaços com dimensão infinita.

Definição 1.13. Sejam $X$ um espaço de Banach, $\Omega \subset X$ um aberto limitado e $T \in C(\bar{\Omega}, X)$ uma aplicação cuja imagem está contida em agum subespaço $F$ de $X$ de dimensão finita. Definimos 
o grau de Leray \& Schauder de $\varphi=I-T$ em relação a $\Omega$ num ponto $b \notin \varphi(\partial \Omega)$, como sendo o número inteiro

$$
\operatorname{deg}(\varphi, \Omega, b)=\operatorname{deg}\left(\left.\varphi\right|_{\bar{\Omega} \cap F}, \Omega \cap F, b\right)
$$

sendo o membro direito da igualdade acima dado pela Definição 1.12.

As funções $\varphi$ da Definição 1.13 são conhecidas como perturbações de dimensão finita da identidade. Nos trabalhos [8] e [9] o leitor encontrará a prova detalhada de que a Definição 1.13 é consistente, isto é, independe da escolha do subespaço $F$.

A partir desse momento, estamos em condições de apresentar o Teorema de Continuação de Mawhin, o qual foi fundamental para obtermos os resultados referentes à existência de soluções periódicas para os problemas que estudamos.

Sejam $X$ e $Y$ espaços de Banach e $W$ um subespaço normado de $X$. Diremos que $L: W \longrightarrow$ $Y$ é um operador de Fredholm se seu núcleo tiver dimensão finita $n$ e sua imagem for um subespaço fechado de $Y$ de codimensão finita $m$. O índice de um operador de Fredholm será a diferença $n-m$. Prova-se que, se $L$ for um operador de Fredholm de índice zero, então existirão operadores lineares contínuos e idempotentes $P: X \longrightarrow X$ e $Q: Y \longrightarrow Y$ satisfazendo as seguintes igualdades

$$
\operatorname{Ker} L=\operatorname{Im} P \quad \text { e } \quad \operatorname{Im} L=\operatorname{Ker} Q .
$$

Se $L$ for um operador de Fredholm, então a primeira igualdade em (1.4) implicará que a restrição de $L$ a $W \cap \operatorname{Ker} P$, que denotamos por $L_{P}: W \cap \operatorname{Ker} P \longrightarrow L(W \cap \operatorname{Ker} P)$, é um isomorfismo. De fato, supondo $\operatorname{Ker} L=\operatorname{Im} P$ e tomando $x \in W \cap \operatorname{Ker} P$ tal que $L_{P}(x)=0$, concluímos que $x \in \operatorname{Im} P$, ou seja, que existe $y \in X$ tal que $P y=x$. Como $P$ é idempotente e $x \in \operatorname{Ker} P$, a última igualdade implica que $x=P y=P x=0$.

Sendo $L: W \longrightarrow Y$ um operador de Fredholm de índice zero e $P, Q$ as projeções men- 
cionadas acima, diremos que um operador contínuo $N: X \longrightarrow Y$ é $L$-compacto no fecho $\bar{\Omega}$ do aberto e limitado $\Omega \subset X$, quando $Q N(\bar{\Omega})$ for limitado e $\left(L_{P}\right)^{-1}(I-Q) N: \bar{\Omega} \longrightarrow X$ for um operador compacto.

Com a notação estabelecida acima, afirmamamos que se $J: \operatorname{Im} Q \longrightarrow \operatorname{Ker} L$ for um isomorfismo, então $J Q N: \operatorname{Ker} L \longrightarrow \operatorname{Ker} L$ será uma perturbação de dimensão finita da identidade. De fato, denotando por $I d$ o operador identidade definido em $\operatorname{Ker} L$, temos $J Q N=I d-(I d-J Q N)$ e a afirmação segue do fato de que $\operatorname{dim} \operatorname{Ker} L<+\infty$.

Ainda mantendo a notação acima, enunciamos o seguinte Teorema de Continuação de Mawhin ([14], p. 40) que desempenha papel fundamental nos resultados sobre existência de solução periódica que obtivemos e que serão apresentados e demonstrados nos dois próximos capítulos.

Teorema 1.14. Sejam $X, Y$ dois espaços de Banach e $W$ um subespaço de $X$. Suponha que $L: W \longrightarrow Y$ seja um operador de Fredholm de índice zero e $N: X \longrightarrow Y$ seja um operador L-compacto em $\bar{\Omega}$, sendo $\Omega$ um subconjunto aberto e limitado de X. Suponha, também, que as seguintes condições sejam satisfeitas:

(i) Se $x \in \partial \Omega \cap W$, então $L x \neq \lambda N x, \lambda \in(0,1)$, em que $\partial \Omega$ denota a fronteira de $\Omega$;

(ii) Se $x \in \partial \Omega \cap \operatorname{Ker} L$, então $Q N x \neq 0$;

(iii) $\operatorname{deg}\{J Q N, \Omega \cap \operatorname{Ker} L, 0\} \neq 0, J Q N: \operatorname{Ker} L \longrightarrow \operatorname{Ker} L$.

Nestas condiçães, a equação Lx $=$ Nx possuirá pelo menos uma solução em $\bar{\Omega} \cap W$. 


\section{Existência e unicidade de solução periódica} para EDFR's impulsivas

\subsection{Introdução}

Neste capítulo, estabeleceremos condições que garantem existência de solução periódica para uma classe de equações diferencias funcionais com retardo sujeitas a condições impulsivas. Além disso, apresentaremos uma condição do tipo Lipschitz e uma condição sobre os momentos de impulso que garantem unicidade de solução periódica.

Os momentos de impulso do problema que estudaremos constituirão uma sequência $\left\{t_{k}\right\}_{k \in \mathbb{N}}$ crescente e ilimitada de números reais positivos. Suporemos que existe uma constante $T>0$ tal que $\left\{t_{k} ; k \in \mathbb{N}\right\} \cap[0, T]=\left\{t_{1}, \ldots t_{m}\right\}$ e, para cada $k \in \mathbb{N}, t_{m+k}=t_{k}+T$. Além disso, $r$ será uma constante dada satisfazendo $0<r \leq T$.

Dado um $I$ intervalo real, uma função $\phi: I \longrightarrow \mathbb{R}$ será dita regrada se, para cada $\tau$ ponto de acumulação à direita de $I$ e para cada $\tau$ ponto de acumulação à esquerda de $I$, os limites laterais $\lim _{t \rightarrow \tau^{+}} \phi(t)$ e $\lim _{t \rightarrow \tau^{-}} \phi(t)$ existirem e forem finitos. Dessa forma, se $a, b \in \mathbb{R}$ com $a<b$, então uma função $\phi:[a, b] \longrightarrow \mathbb{R}$ será dita regrada caso existam e sejam finitos ambos os limites laterais

$$
\lim _{t \rightarrow \tau^{-}} \phi(t), \quad \text { para todo } \tau \in(a, b]
$$




$$
\lim _{t \rightarrow \tau^{+}} \phi(t), \quad \text { para todo } \tau \in[a, b) .
$$

Dados $a, b \in \mathbb{R}$ com $a<b$, denotaremos por $G([a, b], \mathbb{R})$ o espaço de Banach constituído pelo conjunto das funções regradas $\phi:[a, b] \longrightarrow \mathbb{R}$ munido com a norma usual do supremo. Com a notação $G^{-}([a, b], \mathbb{R})$ indicaremos o espaço das funções que pertencem a $G([a, b], \mathbb{R})$ e que são contínuas pela esquerda. Para o leitor interessado em mais detalhes sobre as funções regradas recomendamos os trabalhos [13] e [17].

Dado um número real $t \geq 0$ e uma função $x:[-r,+\infty) \longrightarrow \mathbb{R}$, denotamos por $x_{t}$ a função real dada por $x_{t}(\theta)=x(t+\theta), \theta \in[-r, 0]$. As notações $x\left(t_{k}^{+}\right)$e $\Delta x\left(t_{k}\right)$ representarão, respectivamente, o limite $\lim _{t \rightarrow t_{k}^{+}} x(t)$ e a diferença $x\left(t_{k}^{+}\right)-x\left(t_{k}\right)$.

Nosso objeto de estudo será o problema

$$
\begin{cases}x^{\prime}(t)=f\left(t, x_{t}\right), & t \geq 0, t \neq t_{k}, k \in \mathbb{N} \\ \Delta x\left(t_{k}\right)=I\left(t_{k}, x\left(t_{k}\right)\right), & k \in \mathbb{N} .\end{cases}
$$

Neste problema, $f:[0,+\infty) \times G([-r, 0], \mathbb{R}) \longrightarrow \mathbb{R}$ é uma função $T$-periódica na primeira variável e, para cada $x$ pertencente ao espaço vetorial $G([-r,+\infty), \mathbb{R})$ que seja $T$-periódica em $[0,+\infty)$, a função $[0, T] \ni t \mapsto f\left(t, x_{t}\right)$ pertence a $G^{-}([0, T], \mathbb{R})$. Além disso, $I \in C([0,+\infty) \times$ $\mathbb{R}, \mathbb{R})$.

Definição 2.1. Diremos que uma função $x \in G([-r,+\infty), \mathbb{R})$ é uma solução do problema (2.1), quando

(i) x for diferenciável em cada intervalo $\left(0, t_{1}\right]$ e $\left(t_{k}, t_{k+1}\right], k \in \mathbb{N}$;

(ii) $x$ satisfizer a igualdade $x^{\prime}(t)=f\left(t, x_{t}\right)$, em cada ponto $t \in(0,+\infty) \backslash\left\{t_{k} ; k \in \mathbb{N}\right\}$;

(iii) $\Delta x\left(t_{k}\right)=I\left(t_{k}, x\left(t_{k}\right)\right)$, para cada $k \in \mathbb{N}$. 
Diremos que uma solução do problema (2.1) é T-periódica, quando $x(t)=x(t+T)$ para cada $t \geq 0$.

\subsection{Existência de solução periódica}

Nesta seção, nosso propósito é apresentar condições que garantam a existência de soluções $T$-periódicas para o problema (2.1).

A seguir, apresentamos as hipóteses que, conforme mostraremos, garantem que o problema (2.1) possui pelo menos uma solução $T$-periódica e continuamente diferenciável nos intervalos $\left(0, t_{1}\right],\left(t_{k}, t_{k+1}\right], k \in \mathbb{N}$.

Existe uma constante positiva $d$ tal que

(H1) Se $\varphi \in G([-r, 0], \mathbb{R})$ satisfizer $|\varphi(0)|>d$, então $\varphi(0) f(t, \varphi)>0, t \in[0, T]$;

(H2) Se $|c|>d$, então $c I\left(t_{k}, c\right)>0, \quad k \in\{1, \ldots m\}$.

Além disso,

(H3) Existe $\left(c_{1}, \ldots, c_{m}\right) \in \mathbb{R}^{m}$ satisfazendo $\sum_{k=1}^{m}\left|c_{k}\right|<1$ e tal que $\left|I\left(t_{k}, c\right)\right| \leq\left|c_{k} c\right|, c \in \mathbb{R}, k \in$ $\{1, \ldots m\}$

O resultado principal dessa seção é o teorema seguinte.

Teorema 2.2. Se as condições (H1) a (H3) forem satisfeitas, então o problema (2.1) terá pelo menos uma solução T-periódica continuamente diferenciável nos intervalos $\left(0, t_{1}\right],\left(t_{k}, t_{k+1}\right]$, $k \in \mathbb{N}$.

Visando utilizar o Teorema 1.14 para demonstrar o Teorema 2.2, encontraremos uma equação a operadores $L x=N x$, definida num espaço adequado de funções de tal modo que da existên- 
cia de solução para tal equação possamos inferir que o problema (2.1) possui pelo menos uma solução $T$-periódica de classe $C^{1}$ nos intervalos $\left(0, t_{1}\right],\left(t_{k}, t_{k+1}\right], k \in \mathbb{N}$.

Sejam $X=G([0, T], \mathbb{R})$ e $W$ o subespaço de $X$ formado pelas funções $x \in X$ que satisfazem $x(0)=x(T)$, que são continuamente diferenciáveis nos intervalos $\left(0, t_{1}\right],\left(t_{1}, t_{2}\right], \ldots,\left(t_{m}, T\right]$ e os limites laterais $x^{\prime}\left(t_{k}^{+}\right), k \in\{1, \ldots, m\}$ existem e são finitos. Note que $W$ não é completo e que, de acordo com o Teorema 1.14, o domínio de $L$ deve ser subespaço de um espaço completo. Por essa razão foi que definimos o espaço $X$, que é completo e contém $W$.

Defina $L: W \longrightarrow X \times \mathbb{R}^{m}$, pondo

$$
L x(t)= \begin{cases}\left(x^{\prime}(t),\left(\Delta x\left(t_{1}\right), \ldots, \Delta x\left(t_{m}\right)\right)\right), & t \in(0, T] \backslash\left\{t_{1}, \ldots, t_{m}\right\} \\ \left(x^{\prime}\left(t^{+}\right),\left(\Delta x\left(t_{1}\right), \ldots, \Delta x\left(t_{m}\right)\right)\right), & t=0 \\ \left(x^{\prime}\left(t^{-}\right),\left(\Delta x\left(t_{1}\right), \ldots, \Delta x\left(t_{m}\right)\right)\right), & t=t_{k}, k \in\{1, \ldots, m\}\end{cases}
$$

e considere o operador $N: X \longrightarrow X \times \mathbb{R}^{m}$, dado por

$$
N x(t)=\left(f\left(t, x_{t}\right),\left(I_{1}\left(t_{1}, x\left(t_{1}\right)\right), \ldots, I_{m}\left(t_{m}, x\left(t_{m}\right)\right)\right)\right), \quad t \geq 0,
$$

sendo $f$ e $I$ as funções presentes no problema (3.1). Além disso, consideramos que $x(s)=$ $x(s+T)$ para cada $s \in[-r, 0]$.

Note que se existir uma função $x \in W$ satisfazendo a igualdade

$$
L x=N x
$$

então a extensão $T$-periódica de $x$ a $[-r,+\infty)$ será continuamente diferenciável nos intervalos $\left(0, t_{1}\right],\left(t_{k}, t_{k+1}\right], k \in \mathbb{N}$ e, de acordo com a Definição 2.1 e com as definições de $L$ e de $N$, tal extensão será solução para o problema (2.1).

Dessa forma, com o intuito de demonstrar o Teorema 2.2, nosso objetivo é demonstrar que 
as condições de $(H 1)$ a (H3) implicam que a equação (2.4) possui pelo menos uma solução.

Com os resultados que seguem, mostraremos que estão satisfeitas todas as hipóteses do Teorema 1.14, sendo $L$ e $N$ os operadores definidos em (2.2) e (2.3) respectivamente.

Lema 2.3. O operador L é um operador de Fredholm de índice zero.

Demonstração. Iniciamos a prova mostrando que vale a igualdade

$$
\operatorname{Im} L=\left\{\left(y,\left(a_{1} \ldots, a_{m}\right)\right) \in X \times \mathbb{R}^{m} ; y\left(0^{+}\right)=y(0) \mathrm{e} \int_{0}^{T} y(t) d t+\sum_{k=1}^{m} a_{k}=0\right\}
$$

De fato, seja $\left(y,\left(a_{1}, \ldots, a_{m}\right)\right) \in X \times \mathbb{R}^{m}$ tal que $y\left(0^{+}\right)=y(0)$ e

$$
\int_{0}^{T} y(t) d t+\sum_{k=1}^{m} a_{k}=0
$$

Considere $x:[0, T] \longrightarrow \mathbb{R}$ dada por

$$
x(t)= \begin{cases}0, & t=0 \\ \int_{0}^{t} y(s) d s+\sum_{k=1}^{m} a_{k}, & t \in(0, T] \backslash\left\{t_{1}, \ldots, t_{m}\right\} \\ x\left(t^{+}\right)-a_{k}, & t=t_{k}, k \in\{1, \ldots, m\} .\end{cases}
$$

Então $x \in W$. Vejamos que

$$
L x=\left(y,\left(a_{1}, \ldots, a_{m}\right)\right) .
$$

Se $t \in(0, T] \backslash\left\{t_{1}, \ldots, t_{m}\right\}$, então $x^{\prime}(t)=y(t)$. Portanto,

$$
L x(t)=\left(x^{\prime}(t),\left(\Delta x\left(t_{1}\right), \ldots, \Delta x\left(t_{m}\right)\right)\right)=\left(y(t),\left(a_{1} \ldots, a_{m}\right)\right) \quad t \in(0, T] \backslash\left\{t_{1}, \ldots, t_{m}\right\} .
$$


Por outro lado, utilizando a definição de $x$, obtemos

$$
\begin{aligned}
L x(0) & =\left(x^{\prime}\left(0^{+}\right),\left(\Delta x\left(t_{1}\right), \ldots, \Delta x\left(t_{m}\right)\right)\right)=\left(y\left(0^{+}\right),\left(a_{1}, \ldots, a_{m}\right)\right) \\
& =\left(y(0),\left(a_{1}, \ldots, a_{m}\right)\right) .
\end{aligned}
$$

Por fim, como y é contínua pela esquerda, temos

$$
x^{\prime}\left(t_{k}^{-}\right)=\lim _{t \rightarrow t_{k}^{-}} x^{\prime}(t)=\lim _{t \rightarrow t_{k}^{-}} y(t)=y\left(t_{k}\right) \quad k \in\{1, \ldots, m\} .
$$

Assim, para cada $k \in\{1, \ldots, m\}$, vale

$$
\begin{aligned}
L x\left(t_{k}\right) & =\left(x^{\prime}\left(t_{k}^{-}\right)\left(\Delta x\left(t_{1}\right), \ldots \Delta x\left(t_{m}\right)\right)\right) \\
& =\left(y\left(t_{k}\right)\left(a_{1} \ldots, a_{m}\right)\right)
\end{aligned}
$$

As igualdades obtidas em (2.7), (2.8) e (2.9) implicam que vale a igualdade (2.6) e, portanto,

$$
\left\{\left(y,\left(a_{1} \ldots, a_{m}\right)\right) \in X \times \mathbb{R}^{m} ; y\left(0^{+}\right)=y(0) \text { e } \int_{0}^{T} y(t) d t+\sum_{k=1}^{m} a_{k}=0\right\} \subset \operatorname{Im} L .
$$

Reciprocamente, seja $\left(y,\left(a_{1}, \ldots, a_{m}\right)\right) \in \operatorname{Im} L$. Pela definição de $L$, existe $x \in W$ tal que $x^{\prime}(t)=y(t)$, para $t \in(0, T] \backslash\left\{t_{1}, \ldots, t_{m}\right\}, x^{\prime}\left(0^{+}\right)=y(0)$ e $\Delta x\left(t_{k}\right)=a_{k}, k \in\{1, \ldots, m\}$. Dessa 
forma,

$$
\begin{aligned}
\int_{0}^{T} y(t) d t & =\lim _{\varepsilon \rightarrow 0^{+}} \int_{0}^{t_{1}-\varepsilon} y(t) d t+\lim _{\varepsilon \rightarrow 0^{+}} \int_{t_{1}+\varepsilon}^{t_{2}-\varepsilon} y(t) d t+\ldots+\lim _{\varepsilon \rightarrow 0^{+}} \int_{t_{m}+\varepsilon}^{T-\varepsilon} y(t) d t \\
& =\lim _{\varepsilon \rightarrow 0^{+}} \int_{0}^{t_{1}-\varepsilon} x^{\prime}(t) d t+\lim _{\varepsilon \rightarrow 0^{+}} \int_{t_{1}+\varepsilon}^{t_{2}-\varepsilon} x^{\prime}(t) d t+\ldots+\lim _{\varepsilon \rightarrow 0^{+}} \int_{t_{m}+\varepsilon}^{T-\varepsilon} x^{\prime}(t) d t \\
& =\left[x\left(t_{1}^{-}\right)-x(0)\right]+\left[x\left(t_{2}^{-}\right)-x\left(t_{1}^{+}\right)\right]+\ldots+\left[x\left(T^{-}\right)-x\left(t_{m}^{+}\right)\right] .
\end{aligned}
$$

Como $x$ é contínua pela esquerda e $x(0)=x(T)$, obtemos

$$
\begin{aligned}
\int_{0}^{T} y(t) d t & =\left[x\left(t_{1}\right)-x(0)\right]+\left[x\left(t_{2}\right)-x\left(t_{1}^{+}\right)\right]+\ldots+\left[x(T)-x\left(t_{m}^{+}\right)\right] \\
& =-\sum_{k=1}^{m} \Delta x\left(t_{k}\right)=-\sum_{k=1}^{m} a_{k} .
\end{aligned}
$$

Além disso, como $x^{\prime}\left(0^{+}\right)=y(0)$, então

$$
y\left(0^{+}\right)=\lim _{t \rightarrow 0^{+}} y(t)=\lim _{t \rightarrow 0^{+}} x^{\prime}(t)=x^{\prime}\left(0^{+}\right)=y(0),
$$

o que conclui a demonstração da igualdade (2.5).

Mostremos, agora, que $\operatorname{Im} L$ é fechado em $X \times \mathbb{R}^{m}$. Seja $\left\{\left(y^{n},\left(a_{1}^{n}, \ldots, a_{m}^{n}\right)\right)\right\}_{n \in \mathbb{N}}$ uma sequência em Im $L$ que converge para $\left(y,\left(a_{1}, \ldots, a_{m}\right)\right) \in X \times \mathbb{R}^{m}$. Isto significa que $y^{n} \longrightarrow y$ e $a_{k}^{n} \longrightarrow a_{k}, k \in\{1, \ldots, m\}$. Assim, $y$ é contínua em zero e da igualdade (2.5) e da convergência uniforme de $\left\{y_{n}\right\}$ a $y$, obtemos

$$
\int_{0}^{T} y(t) d t=\int_{0}^{T} \lim _{n \rightarrow+\infty} y^{n}(t) d t=\lim _{n \rightarrow+\infty} \int_{0}^{T} y^{n}(t) d t=-\lim _{n \rightarrow+\infty} \sum_{k=1}^{m} a_{k}^{n}=-\sum_{k=1}^{m} a_{k}
$$


mostrando que $\left(y,\left(a_{1}, \ldots, a_{m}\right)\right) \in \operatorname{Im} L$, como queríamos para concluir que $\operatorname{Im} L$ é fechado em $X \times \mathbb{R}^{m}$.

Para finalizar a prova de que $L$ é um operador de Fredholm de índice zero, resta mostrar que $\operatorname{dim} \operatorname{Ker} L=$ codim $\operatorname{Im} L<\infty$. Da definição de $L$, segue que $\operatorname{Ker} L$ pode ser identificado com $\mathbb{R}$ e, portanto, $\operatorname{dim} \operatorname{Ker} L=1$.

Considere o operador linear $Q$ dado por

$$
Q\left(\left(x,\left(a_{1}, \ldots, a_{m}\right)\right)\right)=\left(\frac{1}{T} \int_{0}^{T} x(t) d t+\frac{1}{T} \sum_{k=1}^{m} a_{k},(0, \ldots, 0)\right),\left(x,\left(a_{1}, \ldots, a_{m}\right)\right) \in X \times \mathbb{R}^{m}
$$

Vejamos que $Q$ é um operador idempotente.

$$
\begin{aligned}
& Q\left(Q\left(\left(x,\left(a_{1}, \ldots, a_{m}\right)\right)\right)\right)=Q\left(\left(\frac{1}{T} \int_{0}^{T} x(t) d t+\frac{1}{T} \sum_{k=1}^{m} a_{k},(0, \ldots, 0)\right)\right) \\
& =\left(\frac{1}{T} \int_{0}^{T}\left[\frac{1}{T} \int_{0}^{T} x(s) d s+\frac{1}{T} \sum_{k=1}^{m} a_{k}\right] d t,(0, \ldots, 0)\right) \\
& =\left(\frac{1}{T} \int_{0}^{T} x(t) d t+\frac{1}{T} \sum_{k=1}^{m} a_{k},(0, \ldots, 0)\right)=Q\left(x,\left(a_{1}, \ldots, a_{m}\right)\right) .
\end{aligned}
$$

Vejamos agora que

$$
\operatorname{Im} Q \cap \operatorname{Ker} Q=\{0\}
$$

Seja

$$
\left(y,\left(b_{1}, \ldots, b_{m}\right)\right) \in \operatorname{Im} Q \cap \operatorname{Ker} Q .
$$

Como $\left(y,\left(b_{1}, \ldots, b_{m}\right)\right) \in \operatorname{Im} Q$, então existem $x \in X$ e $\left(a_{1}, \ldots, a_{m}\right) \in \mathbb{R}^{m}$ tais que $y=\frac{1}{T} \int_{0}^{T} x(t) d t+$ $\frac{1}{T} \sum_{k=1}^{m} a_{k} \mathrm{e}$

$$
b_{1}=b_{2}=\ldots=b_{m}=0 \text {. }
$$


Disso e do fato de que $\left(y,\left(b_{1}, \ldots, b_{m}\right)\right) \in \operatorname{Ker} Q$, obtemos

$$
0=\frac{1}{T} \int_{0}^{T} y(t) d t+\frac{1}{T} \sum_{k=1}^{m} b_{k}=\frac{1}{T} \int_{0}^{T} y(t) d t=y .
$$

De (2.12) e (2.13), obtemos (2.11) e, consequentemente,

$$
X \times \mathbb{R}^{m}=\operatorname{Im} Q \oplus \operatorname{Ker} Q .
$$

Disto, e do fato de que $\operatorname{Im} Q \simeq\{(u,(0, \ldots, 0)) ; u \in \mathbb{R}\}$, podemos inferir que codim $\operatorname{Ker} Q=1$, o que finaliza a demonstração, já que, pela igualdade obtida em (2.5), $\operatorname{Im} L=\operatorname{Ker} Q$.

Nosso objetivo, a seguir, será encontrar um aberto limitado $\Omega \subset X$ que se enquadre nas condições do Teorema 1.14. Faremos isso mediante a obtenção de uma estimativa a priori para soluções $T$-periódicas do problema

$$
\begin{cases}x^{\prime}(t)=\lambda f\left(t, x_{t}\right), & t \geq 0, t \neq t_{k}, k \in \mathbb{N}, \lambda \in(0,1) \\ \Delta x\left(t_{k}\right)=I\left(t_{k}, x\left(t_{k}\right)\right), & k \in \mathbb{N} .\end{cases}
$$

Mais precisamente, após obter uma constante positiva $D$ que majora as possíveis soluções $T$-periódicas do problema (2.14), tomaremos $\Omega$ como o subconjunto de $X$ constituído pelas funções com norma menor que $D$.

Lema 2.4. Se as condições (H1) a (H3) forem satisfeitas, existirá uma constante positiva D, independente de $\lambda$, tal que se $x$ for solução T-periódica do problema (2.14), então $|x(t)| \leq D$, qualquer que seja $t \in[0, T]$.

Demonstração. Suponha que se cumpram as condições $(H 1)$ a $(H 3)$ e que, fixado $\lambda \in(0,1)$, o problema (2.14) possua uma solução $x:[-r,+\infty) \longrightarrow \mathbb{R}$, que seja $T$-periódica. Afirmamos que 
existe $\tau \in[0, T]$, satisfazendo

$$
|x(\tau)| \leq d,
$$

sendo $d$ a constante presente nas hipóteses $(H 1)$ e $(H 2)$. De fato, suponha, por absurdo, que $|x(t)|>d$, para todo $t \in[0, T]$. Então, em particular, $|x(0)|>d$. Se $x(0)>d$, então, pela continuidade de $x$ em $\left[0, t_{1}\right]$, teremos $x(t)>d$ para cada $t \in\left[0, t_{1}\right]$. Além disso, como $x\left(t_{1}\right)>d$, então, pela hipótese $(H 2), I_{1}\left(t_{1}, x\left(t_{1}\right)\right)>0$. Portanto, $x\left(t_{1}^{+}\right)=x\left(t_{1}\right)+I_{1}\left(t_{1}, x\left(t_{1}\right)\right)>d$ e, como $x$ é contínua em $\left(t_{1}, t_{2}\right]$, então $x(t)>d$, para cada $t \in\left(t_{1}, t_{2}\right]$. Seguindo com esse raciocínio, concluímos que $x(t)>d$, qualquer que seja $t \in[0, T]$ ou, equivalentemente, $x_{t}(0)>d$, para cada $t \in[0, T]$. Pela hipótese $(H 1)$, isso implica

$$
f\left(t, x_{t}\right)>0, \quad t \in[0, T]
$$

Por outro lado, como para cada $k \in\{1, \ldots, m\}, x\left(t_{k}\right)>d$, então, pela hipótese $(H 2)$,

$$
I\left(t_{k}, x\left(t_{k}\right)\right)>0, \quad k \in\{1, \ldots, m\} .
$$

De (2.16) e (2.17), obtemos

$$
\lambda \int_{0}^{T} f\left(t, x_{t}\right) d t+\sum_{k=1}^{m} I\left(t_{k}, x\left(t_{k}\right)\right)>0
$$

Do mesmo modo, podemos concluir que, se $x(0)<-d$, então

$$
\lambda \int_{0}^{T} f\left(t, x_{t}\right) d t+\sum_{k=1}^{m} I\left(t_{k}, x\left(t_{k}\right)\right)<0 .
$$

No entanto, nem a desigualdade (2.18) nem a desigualdade (2.19) são verdadeiras, pois inte- 
grando de 0 a $T$ ambos os membros da primeira igualdade do problema (2.14), obtemos

$$
\begin{aligned}
\lambda \int_{0}^{T} f\left(t, x_{t}\right) d t= & \int_{0}^{T} x^{\prime}(t) d t=\left[\lim _{\varepsilon \rightarrow 0+} \int_{0}^{t_{1}-\varepsilon} x^{\prime}(t) d t+\lim _{\varepsilon \rightarrow 0+} \int_{t_{1}+\varepsilon}^{t_{2}-\varepsilon} x^{\prime}(t) d t+\ldots+\right. \\
& \left.+\lim _{\varepsilon \rightarrow 0+} \int_{t_{m-1}+\varepsilon}^{t_{m}-\varepsilon} x^{\prime}(t) d t+\lim _{\varepsilon \rightarrow 0+} \int_{t_{m}+\varepsilon}^{T-\varepsilon} x^{\prime}(t) d t\right] \\
= & x\left(t_{1}^{-}\right)-x(0)+x\left(t_{2}^{-}\right)-x\left(t_{1}^{+}\right)+\ldots+x\left(T^{-}\right)-x\left(t_{m}^{+}\right) .
\end{aligned}
$$

Assim, como $x$ é contínua pela esquerda e $x(0)=x(T)$, temos

$$
\lambda \int_{0}^{T} f\left(t, x_{t}\right) d t=-\sum_{k=1}^{m} I\left(t_{k}, x\left(t_{k}\right)\right) .
$$

Isso conclui a prova de que existe $\tau \in[0, T]$ satisfazendo (2.15).

Como $x$ é contínua pela esquerda, podemos supor, sem perda de generalidade, que $\tau \neq t_{i}, i=$ $1, \ldots, m$. Fixe $t \in(\tau, T]$ e denote por $t_{i_{1}}, \ldots, t_{i_{n}}$ os momentos de impulso entre $\tau$ e $t$. Então

$$
\begin{aligned}
\int_{\tau}^{t} x^{\prime}(s) d s & =\lim _{\varepsilon \rightarrow 0^{+}} \int_{\tau}^{t_{i_{1}-\varepsilon}} x^{\prime}(s) d s+\lim _{\varepsilon \rightarrow 0^{+}} \int_{t_{i_{1}}+\varepsilon}^{t_{i_{2}-\varepsilon}} x^{\prime}(s) d s+\ldots+\lim _{\varepsilon \rightarrow 0^{+}} \int_{t_{i_{n}}+\varepsilon}^{t-\varepsilon} x^{\prime}(s) d s \\
& =x\left(t_{i_{1}}^{-}\right)-x(\tau)+x\left(t_{i_{2}}^{-}\right)-x\left(t_{i_{1}}^{+}\right)+\ldots+x\left(t^{-}\right)-x\left(t_{i_{n}}^{+}\right) \\
& =x\left(t_{i_{1}}\right)-x(\tau)+x\left(t_{i_{2}}\right)-x\left(t_{i_{1}}^{+}\right)+\ldots+x(t)-x\left(t_{i_{n}}^{+}\right) \\
& =x(t)-x(\tau)-\sum_{k=1}^{n} I_{i_{k}}\left(t_{i_{k}}, x\left(t_{i_{k}}\right)\right) .
\end{aligned}
$$


Assim,

$$
\begin{aligned}
|x(t)|-|x(\tau)| & \leq|x(t)-x(\tau)|=\left|\int_{\tau}^{t} x^{\prime}(s) d s+\sum_{k=1}^{n} I_{i_{k}}\left(t_{i_{k}}, x\left(t_{i_{k}}\right)\right)\right| \\
& \leq \int_{0}^{T}\left|x^{\prime}(s)\right| d s+\sum_{k=1}^{n}\left|I_{i_{k}}\left(t_{i_{k}}, x\left(t_{i_{k}}\right)\right)\right|,
\end{aligned}
$$

o que, pela hipótese $(H 3)$, implica

$$
|x(t)|-|x(\tau)| \leq \int_{0}^{T}\left|x^{\prime}(s)\right| d s+\sum_{k=1}^{n}\left|c_{k}\right|\left|x\left(t_{i_{k}}\right)\right|
$$

e, portanto,

$$
\left|x(t) \leq \int_{0}^{T}\right| x^{\prime}(s)\left|d s+\|x\|_{\infty} \sum_{k=1}^{m}\right| c_{k}|+| x(\tau) \mid
$$

Disto, da majoração obtida em (2.15), da primeira equação em (2.14) e do fato de que $\lambda \in(0,1)$, resulta

$$
|x(t)| \leq \int_{0}^{T}\left|f\left(s, x_{s}\right)\right| d s+\|x\|_{\infty} \sum_{k=1}^{m}\left|c_{k}\right|+d
$$

Assim, fixando $R>0$ tal que $\left|f\left(s, x_{s}\right)\right| \leq R, s \in[0, T]$, obtemos

$$
|x(t)| \leq T R+\|x\|_{\infty} \sum_{k=1}^{m}\left|c_{k}\right|+d
$$

Daí, como tomamos $t \in(\tau, T]$ arbitrário, podemos escrever

$$
|x(t)| \leq T R+\|x\|_{\infty} \sum_{k=1}^{m}\left|c_{k}\right|+d, \quad t \in(\tau, T] .
$$

Com o mesmo procedimento, é possível mostrar que

$$
|x(t)| \leq T R+\|x\|_{\infty} \sum_{k=1}^{m}\left|c_{k}\right|+d, \quad t \in[0, \tau) .
$$


Isto e as expressões obtidas em (2.15) e (2.22) implicam

$$
|x(t)| \leq T R+\|x\|_{\infty} \sum_{k=1}^{m}\left|c_{k}\right|+d, \quad t \in[0, T]
$$

Daí, como $\sum_{k=1}^{m}\left|c_{k}\right|<1$, obtemos

$$
\|x\|_{\infty} \leq D:=\frac{T R+d}{1-\sum_{k=1}^{m}\left|c_{k}\right|}
$$

finalizando a demonstração.

A seguir, exibiremos um conjunto aberto e limitado $\Omega \subset X$ tal que o operador $N$ é $L$ compacto no fecho de $\Omega$, que representamos por $\bar{\Omega}$, e tal que se cumprem as condições $(i)$ a (iii) do Teorema 1.14.

Suponha que se cumpram as condições $(H 1)$ até $(H 3)$. Pelo Lema 2.4, existe uma constante $D>0$ tal que se $x \in G([-r,+\infty), \mathbb{R})$ for solução $T$-periódica de (2.14), então $\|x\|_{\infty} \leq D$. Fixe $M>\max \{D, d\}$, sendo $d$ a constante positiva presente nas hipóteses $(H 1)$ e $(H 2)$, e defina o conjunto

$$
\Omega=\left\{x \in X ;\|x\|_{\infty}<M\right\}
$$

Considere os operadores idempotentes $P$ e $Q$ dados por

$$
P: x \mapsto \frac{1}{T} \int_{0}^{T} x(t) d t, x \in X
$$

e

$Q:\left(x,\left(a_{1}, \ldots, a_{m}\right)\right) \mapsto\left(\frac{1}{T} \int_{0}^{T} x(t) d t+\frac{1}{T} \sum_{k=1}^{m} a_{k},(0, \ldots, 0)\right),\left(x,\left(a_{1}, \ldots, a_{m}\right)\right) \in X \times \mathbb{R}^{m}$

É imediato que $\operatorname{Im} P=\operatorname{Ker} L(=\mathbb{R})$ e, pela igualdade (2.5), $\operatorname{Ker} Q=\operatorname{Im} L$. Então os 
operadores $P$ e $Q$ satisfazem as igualdades dadas em (1.4).

Com os dois próximos resultados, mostraremos que o operador $N$ é $L$-compacto em $\bar{\Omega}$. Para tanto, devemos mostrar que $Q N(\bar{\Omega})$ é limitado e que o operador $L_{P}^{-1}(I-Q) N: \bar{\Omega} \longrightarrow W \cap K e r P$ é compacto, sendo $L_{P}$ a restrição de $L$ a $\operatorname{Ker} P$. Iniciamos mostrando que o conjunto $Q N(\bar{\Omega})$ é limitado.

Lema 2.5. Se as condições (H1) a (H3) forem satisfeitas, então o conjunto $Q N(\overline{\mathbf{\Omega}})$ será limitado.

Demonstração. Primeiramente, note que as hipóteses $(H 1)$ a $(H 3)$ garantem a existência do conjunto $\Omega$, como definido em (2.23). Seja $x \in \bar{\Omega}$. Utilizando a norma do máximo $\|\cdot\|_{\max } \mathrm{em}$ $\mathbb{R}^{m+1}$ e a hipótese $(H 3)$, obtemos

$$
\begin{aligned}
\|Q N x\|_{\max } & =\left\|Q\left(f(\cdot, x),\left(I_{1}\left(t_{1}, x\left(t_{1}\right)\right), \ldots, I_{1}\left(t_{1}, x\left(t_{1}\right)\right)\right)\right)\right\|_{\max } \\
& =\left\|\left(\frac{1}{T} \int_{0}^{T} f\left(s, x_{s}\right) d s+\frac{1}{T} \sum_{k=1}^{m} I\left(t_{k}, x\left(t_{k}\right)\right),(0, \ldots 0)\right)\right\|_{\max } \\
& =\frac{1}{T}\left|\int_{0}^{T} f\left(s, x_{s}\right) d s+\sum_{k=1}^{m} I\left(t_{k}, x\left(t_{k}\right)\right)\right| \\
& \leq \frac{1}{T} \int_{0}^{T}\left|f\left(s, x_{s}\right)\right| d s+\frac{1}{T} \sum_{k=1}^{m}\left|I\left(t_{k}, x\left(t_{k}\right)\right)\right| \\
& <R+\frac{M}{T}
\end{aligned}
$$

e a prova está completa.

Para finalizar a prova de que as hipóteses $(H 1)$ a $(H 3)$ implicam que o operador $N$ é $L$ compacto em $\bar{\Omega}$, resta mostrar que tais hipóteses garantem que o operador $L_{P}^{-1}(I-Q) N$ é com- 
pacto. Com o intuito de encontrar $L_{P}^{-1}(I-Q) N$, passamos a buscar a expressão que define a inversa da restrição de $L$ ao núcleo de $P$, a qual denotamos por $L_{P}^{-1}$.

$\operatorname{Seja}\left(y,\left(a_{1}, \ldots, a_{m}\right)\right) \in \operatorname{Im} L_{P}$, devemos encontrar $x \in W \cap \operatorname{Ker} P$ tal que $L_{P} x=\left(y,\left(a_{1}, \ldots, a_{m}\right)\right)$, ou seja,

$$
\left(x^{\prime}(t),\left(\Delta x\left(t_{1}\right), \ldots \Delta x\left(t_{m}\right)\right)\right)=\left(y(t),\left(a_{1}, \ldots, a_{m}\right)\right), \quad t \in[0, T] \backslash\left\{t_{1}, \ldots, t_{m}\right\}
$$

$\mathrm{e}$

$$
\left(x^{\prime}\left(t_{k}^{-}\right),\left(\Delta x\left(t_{1}\right), \ldots \Delta x\left(t_{m}\right)\right)\right)=\left(y\left(t_{k}\right),\left(a_{1}, \ldots, a_{m}\right)\right), \quad k \in\{1, \ldots, m\} .
$$

Observe que, se encontrarmos $x \in W \cap \operatorname{Ker} P$ satisfazendo (2.24), então, como $y$ é contínua pela esquerda, teremos

$$
x^{\prime}\left(t_{k}^{-}\right)=\lim _{t \rightarrow t_{k}^{-}} x^{\prime}(t)=\lim _{t \rightarrow t_{k}^{-}} y(t)=y\left(t_{k}\right), \quad k \in\{1, \ldots, m\}
$$

ou seja, (2.24) implica (2.25). Assim, basta encontrarmos $x \in W \cap \operatorname{Ker} P$ satisfazendo (2.24).

Seja $\hat{x}:[0, T] \longrightarrow \mathbb{R}$ uma função pertencente ao núcleo de $P$, contínua pela esquerda, diferenciável em $(0, T] \backslash\left\{t_{1}, \ldots, t_{m}\right\}$ e tal que

$$
\hat{x}^{\prime}(t)=y(t),[0, T] \backslash\left\{t_{1}, \ldots, t_{m}\right\} \quad \text { e } \quad \Delta \hat{x}\left(t_{k}\right)=a_{k}, k \in\{1, \ldots, m\} .
$$

Encontremos a expressão que define $\hat{x}$. Fixe $t \in[0, T] \backslash\left\{t_{1}, \ldots, t_{m}\right\}$ e denote por $l$ o número de momentos de impulso anteriores a $t$. Cabe salientar que $l$ depende de $t$ e que, por economia de notação, em alguns momentos escreveremos $l$ em vez de $l(t)$. Integrando $y$ de 0 a $t$ e lembrando 
que $\hat{x}$ é contínua pela esquerda, obtemos

$$
\begin{aligned}
\int_{0}^{t} y(s) d s & =\lim _{\varepsilon \rightarrow 0^{+}} \int_{0}^{t_{1}-\varepsilon} \hat{x}^{\prime}(s) d s+\lim _{\varepsilon \rightarrow 0^{+}} \int_{t_{1}+\varepsilon}^{t_{2}-\varepsilon} \hat{x}^{\prime}(s) d s+\ldots+\lim _{\varepsilon \rightarrow 0^{+}} \int_{t_{l}+\varepsilon}^{t} \hat{x}^{\prime}(s) d s \\
& =\left[\hat{x}\left(t_{1}\right)-\hat{x}(0)\right]+\left[\hat{x}\left(t_{2}\right)-\hat{x}\left(t_{1}^{+}\right)\right]+\left[\hat{x}\left(t_{3}\right)-\hat{x}\left(t_{2}^{+}\right)\right]+\ldots+\left[\hat{x}(t)-\hat{x}\left(t_{l}^{+}\right)\right] \\
& =\hat{x}(t)-\hat{x}(0)-\sum_{k=1}^{l} \Delta \hat{x}\left(t_{k}\right) .
\end{aligned}
$$

Dessa forma,

$$
\begin{aligned}
\hat{x}(t) & =\int_{0}^{t} y(s) d s+\sum_{k=1}^{l} \Delta \hat{x}\left(t_{k}\right)+\hat{x}(0) \\
& =\int_{0}^{t} y(s) d s+\sum_{k=1}^{l} a_{k}+\hat{x}(0) .
\end{aligned}
$$

Agora, integrando de 0 a $T$ ambos os membros da última igualdade e levando em conta que $\hat{x}$ pertence ao núcleo de $P$, obtemos

$$
\begin{aligned}
\hat{x}(0) & =-\frac{1}{T} \int_{0}^{T} \int_{0}^{t} y(s) d s d t-\frac{1}{T} \int_{0}^{T} \sum_{k=1}^{l(t)} a_{k} d t \\
& =-\frac{1}{T} \int_{0}^{T} \int_{0}^{t} y(s) d s d t-\sum_{k=1}^{m} a_{k} .
\end{aligned}
$$

Disto e de (2.26), resulta

$$
\hat{x}(t)=\int_{0}^{t} y(s) d s-\frac{1}{T} \int_{0}^{T} \int_{0}^{\tau} y(s) d s d \tau+\sum_{k=1}^{l} a_{k}-\sum_{k=1}^{m} a_{k} .
$$

A seguir, passamos a analizar as três seguintes possibilidades: $t \in\left[0, t_{1}\right), t \in\left(t_{1}, t_{m}\right)$ e $t \in$ $\left(t_{m}, T\right]$. 
Se $t \in\left[0, t_{1}\right)$, então $l=0$ e a expressão obtida em (2.27) se reduz a

$$
\hat{x}(t)=\int_{0}^{t} y(s) d s-\frac{1}{T} \int_{0}^{T} \int_{0}^{\tau} y(s) d s d \tau-\sum_{k=1}^{m} a_{k}
$$

Se $t \in\left(t_{1}, t_{m}\right)$, então $1 \leq l \leq m-1$ e obtemos, a partir da igualdade (2.27), a expressão

$$
\hat{x}(t)=\int_{0}^{t} y(s) d s-\frac{1}{T} \int_{0}^{T} \int_{0}^{\tau} y(s) d s d \tau-\sum_{k=l+1}^{m} a_{k}
$$

Note que, ao supormos $t \in\left(t_{1}, t_{m}\right)$, estamos supondo $m>1$. Por outro lado, observando a expressão obtida em (2.27), notamos que se $m=1$, então

$$
\hat{x}(t)=\int_{0}^{t} y(s) d s-\frac{1}{T} \int_{0}^{T} \int_{0}^{\tau} y(s) d s d \tau
$$

que é também a expressão para $\hat{x}$ nos pontos $t \in\left(t_{m}, T\right]$, para $m \geq 1$, caso em que $l=m$.

Como tomamos $t \in[0, T] \backslash\left\{t_{1}, \ldots, t_{m}\right\}$ arbitrário, então, das expressões obtidas em (2.28) a (2.30), podemos concluir que

$$
\hat{x}(t)=\int_{0}^{t} y(s) d s-\frac{1}{T} \int_{0}^{T} \int_{0}^{\tau} y(s) d s d \tau-\sum_{k=l(t)+1}^{m} a_{k}, \quad t \in[0, T] \backslash\left\{t_{1}, \ldots, t_{m}\right\},
$$

sendo nulo o somatório acima quando $l=m$.

Isso nos motiva a definir a função

$$
x(t)=\int_{0}^{t} y(s) d s-\frac{1}{T} \int_{0}^{T} \int_{0}^{\tau} y(s) d s d \tau-\sum_{k=l(t)+1}^{m} a_{k}, \quad t \in[0, T]
$$

Então, de (2.31), obtemos

$$
x^{\prime}(t)=y(t), \quad t \in[0, T] \backslash\left\{t_{1}, \ldots, t_{m}\right\} .
$$


Vejamos que $x\left(t_{i}^{+}\right)-x\left(t_{i}\right)=a_{i}, i \in\{1, \ldots, m\}$. Fixe $i \in\{1, \ldots, m\}$. Para cada $t>t_{i}$ suficientemente próximo de $t_{i}$, o número de momentos de impulso anteriores a $t$ é $l(t)=i$. Então,

$$
\begin{aligned}
x\left(t_{i}^{+}\right) & =\lim _{t \rightarrow t_{i}^{+}} x(t)=\lim _{t \rightarrow t_{i}^{+}}\left[\int_{0}^{t} y(s) d s-\frac{1}{T} \int_{0}^{T} \int_{0}^{\tau} y(s) d s d \tau-\sum_{k=i+1}^{m} a_{k}\right] \\
& =\int_{0}^{t_{i}} y(s) d s-\frac{1}{T} \int_{0}^{T} \int_{0}^{\tau} y(s) d s d \tau-\sum_{k=i+1}^{m} a_{k} .
\end{aligned}
$$

Por outro lado,

$$
x\left(t_{i}\right)=\int_{0}^{t_{i}} y(s) d s-\frac{1}{T} \int_{0}^{T} \int_{0}^{\tau} y(s) d s d \tau-\sum_{k=i}^{m} a_{k}
$$

Assim,

$$
x\left(t_{i}^{+}\right)-x\left(t_{i}\right)=a_{i}
$$

como queríamos.

Além disso, como $l(t)=m$ para cada $t \in\left(t_{m}, T\right]$, então

$$
\begin{aligned}
\int_{0}^{T} x(t) d t & =\int_{0}^{T} \int_{0}^{t} y(s) d s d t-\frac{1}{T} \int_{0}^{T} \int_{0}^{T} \int_{0}^{\tau} y(s) d s d \tau d t-\int_{0}^{T} \sum_{l(t)+1}^{m} a_{k} d t \\
& =\int_{0}^{T} \int_{0}^{t} y(s) d s d t-\int_{0}^{T} \int_{0}^{\tau} y(s) d s d \tau=0 .
\end{aligned}
$$

As igualdades obtidas em (2.33) a (2.35) nos permitem concluir que a função $x \in W \cap \operatorname{Ker} P$, que buscamos, satisfazendo (2.25), é a função $x$ definida em (2.32). Dessa forma, podemos concluir que, para cada $t \in[0, T]$, temos

$$
L_{p}^{-1}\left(y,\left(a_{1}, \ldots, a_{m}\right)\right)(t)=x(t)=\int_{0}^{t} y(s) d s-\frac{1}{T} \int_{0}^{T} \int_{0}^{\tau} y(s) d s d \tau-\sum_{k=l(t)+1}^{m} a_{k}
$$

Encontremos a expressão que define $L_{P}^{-1}(I-Q) N$ para, na sequência, provar que tal opera- 
dor é compacto no fecho $\bar{\Omega}$ de $\Omega$. Usando as expressões que definem $N$ e $P$, obtemos

$$
\begin{gathered}
L_{P}^{-1}(I-Q) N x=L_{P}^{-1}(I-Q)\left(f(\cdot, x .),\left(I_{1}\left(t_{1}, x\left(t_{1}\right)\right), \ldots, I_{m}\left(t_{m}, x\left(t_{m}\right)\right)\right)=\right. \\
=L_{P}^{-1}\left(f(\cdot, x .)-\frac{1}{T} \int_{0}^{T} f\left(w, x_{w}\right) d w-\frac{1}{T} \sum_{k=1}^{m} I\left(t_{k}, x\left(t_{k}\right)\right),\right. \\
\left.\left(I_{1}\left(t_{1}, x\left(t_{1}\right), \ldots, I_{m}\left(t_{m}, x\left(t_{m}\right)\right)\right)\right)\right) .
\end{gathered}
$$

Assim, pela expressão para $L_{P}^{-1}$, obtida em (2.36), temos

$$
\begin{gathered}
L_{P}^{-1}(I-Q) N x(t)=\int_{0}^{t} f\left(s, x_{s}\right) d s-\frac{t}{T} \int_{0}^{T} f\left(w, x_{w}\right) d w-\frac{t}{T} \sum_{k=1}^{m} I\left(t_{k}, x\left(t_{k}\right)\right) \\
-\frac{1}{T} \int_{0}^{T} \int_{0}^{\tau} f\left(s, x_{s}\right) d s d \tau+\frac{1}{T^{2}} \int_{0}^{T} \int_{0}^{\tau} \int_{0}^{T} f\left(w, x_{w}\right) d w d s d \tau \\
-\sum_{k=l(t)+1}^{m} I\left(t_{k}, x\left(t_{k}\right)\right)+\frac{1}{T^{2}} \sum_{k=1}^{m} I\left(t_{k}, x\left(t_{k}\right)\right) \int_{0}^{T} \int_{0}^{\tau} d s d \tau
\end{gathered}
$$

sendo $l(t)$ o número de momentos de impulso anteriores a $t$. Assim,

$$
\begin{gathered}
L_{P}^{-1}(I-Q) N x(t)=\int_{0}^{t} f\left(s, x_{s}\right) d s-\frac{t}{T} \int_{0}^{T} f\left(s, x_{s}\right) d s-\frac{t}{T} \sum_{k=1}^{m} I\left(t_{k}, x\left(t_{k}\right)\right) \\
-\frac{1}{T} \int_{0}^{T} \int_{0}^{\tau} f\left(s, x_{s}\right) d s d \tau+\frac{1}{2} \int_{0}^{T} f\left(s, x_{s}\right) d s+\frac{1}{2} \sum_{k=1}^{m} I\left(t_{k}, x\left(t_{k}\right)\right)-\sum_{k=l(t)+1}^{m} I\left(t_{k}, x\left(t_{k}\right)\right) .
\end{gathered}
$$

Para provar que o operador $L_{P}^{-1}(I-Q) N$ é compacto, e assim finalizarmos a prova de que $N$ é $L$-compacto em $\bar{\Omega}$, necessitamos expor o conceito de espaço equirregrado.

Definição 2.6. Sejam $a, b \in \mathbb{R}$ tais que $a<b$. Diremos que um conjunto $E \subset G([a, b], \mathbb{R})$ tem limites laterais uniformes num ponto $\xi \in[a, b]$ se, para cada $\varepsilon>0$, existir $\delta>0$ tal que para cada $y \in E$, valerem as implicações seguintes:

(i) Se $t \in[a, b] e \xi<t<\xi+\delta$, então $\left|y(t)-y\left(\xi^{+}\right)\right|<\varepsilon$; 
(ii) Se $t \in[a, b] e \xi-\delta<t<\xi$, então $\left|y(t)-y\left(\xi^{-}\right)\right|<\varepsilon$.

Um conjunto $E \subset G([a, b], \mathbb{R})$ será chamado de equirregrado, quando tiver limites laterais uniformes em todos os pontos $\tau \in[a, b]$.

O próximo resultado será importante na demonstração de que o operador $L_{P}^{-1}(I-Q) N$ é compacto.

Lema 2.7. Um conjunto $E \subset G([a, b], \mathbb{R})$ será relativamente compacto se, e somente se, for equirregrado e, para cada $t \in[a, b]$, o conjunto $\{y(t) ; y \in E\}$ for limitado em $\mathbb{R}$.

Demonstração. Ver [13].

Juntamente com o Lema 2.5, o próximo resultado implica que, se as condições $(H 1)$ a $(H 3)$ forem satisfeitas, então o operador $N$ será $L$-compacto em $\bar{\Omega}$.

Lema 2.8. Se as condições (H1) a (H3) forem satisfeitas, então o operador $L_{P}^{-1}(I-Q) N$ : $\bar{\Omega} \longrightarrow W \cap$ Ker P será compacto.

Demonstração. Suponha que as condições $(H 1)$ até $(H 3)$ sejam satisfeitas. Pelo Lema 2.4, existe o conjunto $\Omega$ definido em (2.23). Seja $\Lambda \subset \bar{\Omega}$. Para provar o resultado enunciado devemos mostrar que o conjunto $E=L_{P}^{-1}(I-Q) N(\Lambda)$ é relativamente compacto. Para tanto, utilizaremos o Lema 2.7.

Iniciamos mostrando que $E$ é equirregular. Fixe $\xi \in[0, T]$ e $\varepsilon>0$. Devemos mostrar que existe $\delta>0$ tal que para cada $y \in E$ as condições seguintes sejam satisfeitas:

$$
t \in[0, T], \quad \xi<t<\xi+\delta \Longrightarrow\left|y(t)-y\left(\xi^{+}\right)\right|<\varepsilon
$$

$\mathrm{e}$

$$
t \in[0, T], \quad \xi-\delta<t<\xi \Longrightarrow\left|y(t)-y\left(\xi^{-}\right)\right|<\varepsilon
$$


Provemos que vale (2.38). A prova de (2.39) pode ser feita seguindo os mesmos procedimentos. Seja $y \in E$. Então existe $x \in \Lambda$ tal que $y(t)=L_{P}^{-1}(I-Q) N x(t)$ para cada $t \in[0, T]$. Dessa forma,

$$
y\left(\xi^{+}\right)=\lim _{t \rightarrow \xi^{+}} y(t)=\lim _{t \rightarrow \xi^{+}} L_{P}^{-1}(I-Q) N x(t) .
$$

Para encontrar o limite indicado acima separaremos a demonstração em dois casos possíveis: considerando o caso em que $\xi$ é um momento de impulso e, posteriormente, supondo o contrário.

Suponha que exista $j \in\{1, \ldots, m\}$ tal que $\xi=t_{j}$. Note que, para cada $t>\xi$ suficientemente próximo de $\xi$, o número de momentos de impulso anteriores a $t$ é $l(t)=j$. Dessa forma, a função $t \mapsto l(t)$, definida numa vizinhança suficientemente pequena à direita de $\xi$, é constante igual a $j$. Portanto $l(t) \rightarrow j$, quando $t$ tende a $\xi$ pela direita. Assim, usando as expressões obtidas em (2.37) e (2.40), obtemos

$$
\begin{aligned}
y\left(\xi^{+}\right)= & \int_{0}^{\xi} f\left(s, x_{s}\right) d s-\frac{\xi}{T} \int_{0}^{T} f\left(s, x_{s}\right) d s-\frac{\xi}{T} \sum_{k=1}^{m} I\left(t_{k}, x\left(t_{k}\right)\right)-\frac{1}{T} \int_{0}^{T} \int_{0}^{\tau} f\left(s, x_{s}\right) d s d \tau \\
& +\frac{1}{2} \int_{0}^{T} f\left(s, x_{s}\right) d s+\frac{1}{2} \sum_{k=1}^{m} I\left(t_{k}, x\left(t_{k}\right)\right)-\sum_{k=j+1}^{m} I\left(t_{k}, x\left(t_{k}\right)\right) .
\end{aligned}
$$

Disto e de (2.37), podemos concluir que se $t>\xi$ estiver suficientemente próximo de $\xi$, então

$$
\left|y(t)-y\left(\xi^{+}\right)\right| \leq \int_{\xi}^{t}\left|f\left(s, x_{s}\right)\right| d s+\frac{t-\xi}{T} \int_{0}^{T}\left|f\left(s, x_{s}\right)\right| d s+\frac{t-\xi}{T} \sum_{k=1}^{m}\left|I\left(t_{k}, x\left(t_{k}\right)\right)\right| .
$$

Daí, como $\left|f\left(s, x_{s}\right)\right| \leq R$ para cada $s \in[0, T],\left|x\left(t_{k}\right)\right| \leq M$ e como, pela hipótese (H3), $\left|I\left(t_{k}, x\left(t_{k}\right)\right)\right| \leq\left|c_{k}\right|\left|x\left(t_{k}\right)\right|$ e $\sum_{k=1}^{m}\left|c_{k}\right|<1$, então

$$
\left|y(t)-y\left(\xi^{+}\right)\right| \leq 2 R(t-\xi)+\frac{M}{T}(t-\xi) .
$$


Assim, para obter (2.38), basta tomar $\delta<\varepsilon /(2 R+M / T)$ suficientemente pequeno, de modo que $l(t)=j$ para cada $t \in[0, T]$ tal que $\xi<t<\xi+\delta$.

A prova de (2.39), supondo que $\xi$ não é um momento de impulso não apresenta nenhuma dificuldade adicional, bastando seguir o mesmo raciocínio empregado acima.

De acordo com o Lema 2.7, para finalizar a demonstração, resta mostrar que para cada $t \in[0, T]$ o conjunto $\{y(t) ; y \in E\}$ é limitado em $\mathbb{R}$.

Sejam $y \in E$ e $x \in \Lambda$ tal que $y=L_{P}^{-1}(I-Q) N x$. Fixe $t \in[0, T]$. Utilizando a expressão obtida em (2.37) e a desigualdade triangular, podemos concluir que

$$
\begin{aligned}
|y(t)| \leq & \int_{0}^{t}\left|f\left(s, x_{s}\right)\right| d s+\frac{t}{T} \int_{0}^{T}\left|f\left(s, x_{s}\right)\right| d s+\frac{t}{T} \sum_{k=1}^{m}\left|I\left(t_{k}, x\left(t_{k}\right)\right)\right|+\frac{1}{T} \int_{0}^{T} \int_{0}^{\tau}\left|f\left(s, x_{s}\right)\right| d s d \tau \\
& +\frac{1}{2} \int_{0}^{T}|f(s, x(s))| d s+\frac{1}{2} \sum_{k=1}^{m}\left|I\left(t_{k}, x\left(t_{k}\right)\right)\right|+\sum_{k=l(t)+1}^{m}\left|I\left(t_{k}, x\left(t_{k}\right)\right)\right| .
\end{aligned}
$$

Portanto,

$$
|y(t)| \leq 2 t R+\frac{t M}{T}+T R+\frac{3 M}{2} \leq 3 T R+\frac{5 M}{2}
$$

e o resultado está demonstrado.

Já mostramos que o operador $L$ é de Fredholm de índice zero e que as hipóteses $(H 1)$ a (H3) implicam que o operador $N$ é $L$-compacto em $\bar{\Omega}$. Assim, para concluirmos que a equação $L x=N x$ possui solução, e que, portanto, o problema (2.1) possui solução $T$-periódica e de classe $C^{1}$ nos intervalos $\left(0, t_{1}\right],\left(t_{k}, t_{k+1}\right], k \in \mathbb{N}$, basta mostrarmos que se $(H 1)$ a $(H 3)$ forem satisfeitas, então se cumprirão as condições $(i),(i i)$ e (iii) do Teorema 1.14. É o que faremos a seguir. 
Lema 2.9. Se as condições (H1) até (H3) forem satisfeitas, então se cumprirão as condições seguintes:

(i) Se $x \in W \cap \partial \Omega$, então $L x \neq \lambda N x$, qualquer que seja $\lambda \in(0,1)$;

(ii) Se $x \in \operatorname{Ker} L \cap \partial \Omega$, então $Q N x \neq 0$;

(iii) $\operatorname{deg}\{J Q N, \Omega \cap \operatorname{Ker} L, 0\} \neq 0$, sendo J :Im $Q \longrightarrow$ Ker L um isomorfismo.

Demonstração. Suponha que se cumpram as condições $(H 1)$ a (H3). Se $x \in W \cap \partial \Omega$, então $\|x\|_{\infty}=M>D$ e, pela estimativa a priori dada pelo Lema 2.4, teremos $L x \neq \lambda N x$, para todo $\lambda \in(0,1)$, e o item $(i)$ está provado.

Provemos (ii). Seja $x \in \operatorname{Ker} L \cap \partial \Omega$. Então ou $x \equiv M$ ou $x \equiv-M$. Assim, ou $x_{t}(0)=M$, para cada $t \in[0, T]$ ou $x_{t}(0)=-M$ para cada $t \in[0, T]$.

Se valer o primeiro caso, então, como $M>d$, por $(H 1)$ e $(H 2)$, teremos

$$
\frac{1}{T} \int_{0}^{T} f\left(t, x_{t}\right) d t+\frac{1}{T} \sum_{k=1}^{m} I\left(t_{k}, x\left(t_{k}\right)\right)>0
$$

e, por conseguinte,

$$
Q N x=\left(\frac{1}{T} \int_{0}^{T} f\left(t, x_{t}\right) d t+\frac{1}{T} \sum_{k=1}^{m} I\left(t_{k}, x\left(t_{k}\right)\right),(0, \ldots 0)\right) \neq 0 .
$$

Do mesmo modo, se valer o segundo caso, isto é, se $x \equiv-M$, então, de $(H 1)$ e $(H 2)$, obteremos

$$
Q N x=\frac{1}{T} \int_{0}^{T} f\left(t, x_{t}\right) d t+\frac{1}{T} \sum_{k=1}^{m} I\left(t_{k}, x\left(t_{k}\right)\right)<0
$$

e, consequentemente,

$$
Q N x=\left(\frac{1}{T} \int_{0}^{T} f\left(t, x_{t}\right) d t+\frac{1}{T} \sum_{k=1}^{m} I\left(t_{k}, x\left(t_{k}\right)\right),(0, \ldots 0)\right) \neq 0 .
$$


Finalizando a prova de que a condição $(i i)$ é satisfeita.

Por fim, vejamos que se cumpre a condição $(i i i)$. Considere o isomorfismo $J: \operatorname{Im} Q \longrightarrow$ $\operatorname{Ker} L, J(x,(0, \ldots, 0))=x$. Para mostrar que $\operatorname{deg}_{B}\{J Q N, \Omega \cap \operatorname{Ker} L, 0\}$ tem sentido, vejamos que $0 \notin J Q N(\partial(\Omega \cap \operatorname{Ker}(L)))$.

Note que $\Omega \cap \operatorname{Ker} L=(-M, M) \subset \mathbb{R}$ e, consequentemente, a fronteira de $\Omega \cap \operatorname{Ker} L$, que denotamos por $\partial(\Omega \cap \operatorname{Ker} L)$, coincide com $\{-M, M\}$. Então, da definição de $Q, N$ e $J$ e da desigualdade (2.41), resulta

$$
\begin{aligned}
J Q N(M) & =J Q\left(f(\cdot, M),\left(I_{1}\left(t_{1}, M\right)\right), \ldots, I_{m}\left(t_{m}, M\right)\right) \\
& =J\left(\left(\frac{1}{T} \int_{0}^{T} f(t, M) d t+\frac{1}{T} \sum_{k=1}^{m} I\left(t_{k}, M\right),(0, \ldots, 0)\right)\right) \\
& \left.=\frac{1}{T} \int_{0}^{T} f(t, M) d t+\frac{1}{T} \sum_{k=1}^{m} I\left(t_{k}, M\right)\right)>0 .
\end{aligned}
$$

Analogamente, da desigualdade (2.42) obtemos $J Q N(-M)<0$.

Para provar o item (iii), ou seja, $\operatorname{deg}_{B}\{J Q N, \Omega \cap \operatorname{Ker} L, 0\} \neq 0$, utilizaremos a propriedade da invariância do grau por homotopia. Considere a aplicação $H:(\bar{\Omega} \cap \operatorname{Ker} L) \times[0,1] \longrightarrow \mathbb{R}$ dada por

$$
H(s, \lambda)=(1-\lambda) s+\frac{\lambda}{T} \int_{0}^{T} f(t, s) d t+\frac{\lambda}{T} \sum_{k=1}^{m} I\left(t_{k}, s\right) .
$$

Como $M>d$, então, pela hipóteses $(H 1)$ e $(H 2)$, podemos concluir que para cada $\lambda \in[0,1]$, vale

$$
H(M, \lambda)=(1-\lambda) M+\frac{\lambda}{T} \int_{0}^{T} f(t, M) d t+\frac{\lambda}{T} \sum_{k=1}^{m} I\left(t_{k}, M\right)>0
$$

$\mathrm{e}$

$$
H(-M, \lambda)=(1-\lambda)(-M)+\frac{\lambda}{T} \int_{0}^{T} f(t,-M) d t+\frac{\lambda}{T} \sum_{k=1}^{m} I\left(t_{k},-M\right)<0 .
$$


Assim, $0 \notin H(\cdot, 1)(\{-M, M\})$, bem como $0 \notin H(\cdot, 0)(\{-M, M\})$. Portanto têm sentido as expressões $\operatorname{deg}\{H(\cdot, 1), \Omega \cap \operatorname{KerL}, 0\}$ e $\operatorname{deg}\{H(\cdot, 0), \Omega \cap \operatorname{KerL}, 0\}$.

Fixando $\lambda=0$ na expressão que define $H$, obtemos $H(\cdot, 0)=I d$. Disto e da invariância do grau por homotopia, obtemos

$$
\operatorname{deg}\{H(\cdot, 1), \Omega \cap \operatorname{KerL}, 0\}=\operatorname{deg}\{H(\cdot, 0), \Omega \cap \operatorname{KerL}, 0\}=1 .
$$

Por outro lado, para cada $s \in \Omega \cap \operatorname{Ker} L$, vale

$$
H(s, 1)=\frac{1}{T} \int_{0}^{T} f(t, s) d t+\frac{1}{T} \sum_{k=1}^{m} I\left(t_{k}, s\right)=J Q N s
$$

De (2.43) e (2.44), obtemos

$$
\operatorname{deg}\{J Q N, \Omega \cap \operatorname{KerL}, 0\}=1,
$$

finalizando a demonstração.

Neste momento, estamos em condições de demonstrar o Teorema 2.2, que é o resultado principal desta seção.

Demonstração. (do Teorema 2.2) Suponha que as condições (H1) a (H3) estejam satisfeitas. Pelo Teorema 1.14, Lema 2.3 e Lemas 2.5 a 2.9, podemos concluir que a equação $L x=N x$ possui pelo menos uma solução $x:[0, T] \longrightarrow \mathbb{R}$ pertencente a $W \cap \bar{\Omega}$. Dessa forma, ao extender $x$, de forma $T$-periódica, a $[-r,+\infty)$ obtemos uma função que possui todas as propriedades enunciadas no Teorema 2.2 e, pela Definição 2.1, tal extensão é solução do problema (2.1). 


\subsection{Unicidade de solução periódica}

O resultado obtido nesta seção foi inspirado em parte do trabalho [31], no qual os autores obtiveram condições suficientes para a unicidade de solução para o problema autônomo não impulsivo

$$
\left\{\begin{array}{l}
x^{\prime}(t)=g\left(x_{t}\right), \quad t_{0} \leq t \leq t_{0}+\delta \\
x\left(t_{0}\right)=\varphi_{0},
\end{array}\right.
$$

no qual são dados $g: C([-M, 0], \mathbb{R}) \longrightarrow \mathbb{R}, t_{0} \in \mathbb{R}, \delta>0$ e $\varphi_{0} \in C([-M, 0], \mathbb{R})$, sendo $M$ uma constante positiva fixada.

Aqui, mostraremos que se a função $f$, do problema impulsivo (2.1), satisfizer uma certa condição do tipo Lipschitz e se o último momento de impulso menor que $T$ distar deste mais que $r$, então o problema (2.1) não possuirá duas soluções $T$-periódicas distintas coincidindo em $[-r, \sigma]$, seja qual for $\sigma<T$ que diste mais que $r$ do último momento de impulso e seja maior que o mesmo.

A condição sobre $f$, mencionada acima é a seguinte:

(H) Para cada $\xi \in G([-r, 0], \mathbb{R})$, existem $\delta=\delta(\xi)>0$ e $L=L(\delta)>0$ tais que se $\varphi, \psi \in$ $G([-r, 0], \mathbb{R})$ forem tais que $\|\varphi-\xi\|_{\infty}<\delta$ e $\|\psi-\xi\|_{\infty}<\delta$, então

$$
|f(t, \varphi)-f(t, \psi)| \leq L|\varphi(0)-\psi(0)|, \quad t \in[0, T] .
$$

Teorema 2.10. Se a condição $(H)$ for satisfeita e se $t_{m}+r<T$ então o problema (2.1) não possuirá duas soluções $T$-periódicas distintas coincidindo em $[-r, \sigma]$, se $t_{m}+r<\sigma<T$.

Demonstração. Suponha que se cumpra a condição $(H)$ e que $t_{m}+r<T$. Fixe $\sigma \in\left(t_{m}+r, T\right)$. Suponha que $x:[-r,+\infty) \longrightarrow \mathbb{R}$ e $y:[-r,+\infty) \longrightarrow \mathbb{R}$ sejam soluções $T$-periódicas de (2.1) que coincidem em $[-r, \sigma]$. Mostraremos que $x \equiv y$. 
Defina o conjunto

$$
A=\{t \in[0, T] ; x(s)=y(s) \text { para } 0 \leq s \leq t\}
$$

Uma vez que $x$ e $y$ são $T$-periódicas, então, para concluirmos que $x \equiv y$, basta mostrarmos que $T \in A$. Faremos isso mediante uma prova indireta. Mais precisamente, mostraremos que se $T \notin A$, então existirá $\eta>0$ tal que $\sup A+\eta \in A$. Antes disso, vejamos que $A$ possui supremo e que o mesmo pertence a $A$.

Como $A$ é limitado superiormente e é não vazio, pois $\sigma \in A$, então existe $\sup A$. A seguir, mostraremos que $A$ é um conjunto compacto para concluir que $\sup A \in A$. Para tanto, basta mostrar que tal conjunto é fechado, pois é um subconjunto limitado de $\mathbb{R}$.

Seja $\left\{t_{n}\right\}$ uma sequência em $A$ convergindo para um ponto $t_{0}$. Vejamos que $t_{0} \in A$. Se existir algum $n \in \mathbb{N}$ tal que $t_{0}<t_{n}$, então, da definição do conjunto $A$, segue que $x(s)=y(s)$, para cada $s \in\left[0, t_{0}\right]$, mostrando que $t_{0} \in A$, como queríamos. Suponha, agora, que para cada $n \in \mathbb{N}, t_{n}<t_{0}$. Para cada $\rho<t_{0}$ existe $n \in \mathbb{N}$ tal que $\rho<t_{n}$ e, como $t_{n} \in A$, então $\rho \in A$. Assim,

$$
\rho<t_{0} \Longrightarrow x(s)=y(s), \quad s \in[0, \rho]
$$

Por outro lado, como $x$ e $y$ são contínuas pela esquerda em $t_{0}$, então $x\left(t_{n}\right) \longrightarrow x\left(t_{0}\right)$ e $y\left(t_{n}\right) \longrightarrow$ $y\left(t_{0}\right)$. Portanto, como $x\left(t_{n}\right)=y\left(t_{n}\right)$, para cada $n \in \mathbb{N}$, temos $x\left(t_{0}\right)=y\left(t_{0}\right)$. Disto e de (2.46), concluímos que $t_{0} \in A$, encerrando a prova de que $A$ é fechado. Logo $A$ é compacto e $\sup A \in A$.

Como observamos acima, nosso objetivo é demonstrar que $T \in A$. Suponha, por absurdo, que $T \notin A$. Então, denotando $\tau:=\sup A$, obtemos

$$
t_{m}+r<\tau<T
$$


Note que a primeira desigualdade acima resulta de $t_{m}+r<\sigma \in A$.

Como $\tau \in A$ e, além disso, $x$ e $y$ coincidem em $[-r, 0]$, então

$$
z_{\tau}:=x_{\tau}=y_{\tau}
$$

Além disso, por $(H)$, existem $\delta>0$ e $L=L(\delta)>0$ tais que, para cada $t \in[0, T]$, vale a implicação

$\varphi, \psi \in G([-r, 0], \mathbb{R}),\left\|\varphi-z_{\tau}\right\|_{\infty}<\delta,\left\|\psi-z_{\tau}\right\|_{\infty}<\delta \Longrightarrow|f(t, \varphi)-f(t, \psi)| \leq L|\varphi(0)-\psi(0)|$

Afirmamos que existe $\eta, 0<\eta \leq T-\tau$, tal que

$$
t \in[\tau, \tau+\eta] \Longrightarrow\left\|x_{t}-x_{\tau}\right\|_{\infty}<\delta \quad \text { e } \quad t \in[\tau, \tau+\eta] \Longrightarrow\left\|y_{t}-y_{\tau}\right\|_{\infty}<\delta
$$

Com efeito, como $t_{m}<\tau-r$, podemos concluir que a função $x$ é uniformemente contínua em $[\tau-r, T]$. Portanto existe $\eta_{1}, 0<\eta_{1} \leq T-\tau$, tal que

$$
p, q \in[\tau-r, T],|p-q| \leq \eta_{1} \Longrightarrow|x(p)-x(q)|<\delta
$$

Fixe $t \in\left[\tau, \tau+\eta_{1}\right]$. Então, para cada $\theta \in[-r, 0]$, temos

$$
t+\theta, \tau+\theta \in[\tau-r, T] \quad \text { e } \quad|(t+\theta)-(\tau+\theta)| \leq \eta_{1}
$$

Disto e de (2.50), segue que

$$
t \in\left[\tau, \tau+\eta_{1}\right] \Longrightarrow|x(t+\theta)-x(\tau+\theta)|<\delta,
$$


qualquer que seja $\theta \in[-r, 0]$. Em particular,

$$
t \in\left[\tau, \tau+\eta_{1}\right] \Longrightarrow \max _{\theta \in[-r, 0]}|x(t+\theta)-x(\tau+\theta)|<\delta,
$$

ou seja,

$$
t \in\left[\tau, \tau+\eta_{1}\right] \Longrightarrow\left\|x_{t}-x_{\tau}\right\|_{\infty}<\delta
$$

Seguindo o mesmo raciocínio, é possível mostrar que existe $\eta_{2}, 0<\eta_{2} \leq T-\tau$, tal que

$$
t \in\left[\tau, \tau+\eta_{2}\right] \Longrightarrow\left\|y_{t}-y_{\tau}\right\|_{\infty}<\delta
$$

Assim, tomando $\eta=\min \left\{\eta_{1}, \eta_{2}\right\}$, obtemos (2.49).

De (2.47), (2.48) e (2.49), resulta

$$
t \in[\tau, \tau+\eta] \Longrightarrow\left|f\left(t, x_{t}\right)-f\left(t, y_{t}\right)\right| \leq L|x(t)-y(t)|
$$

Dessa forma, fixando $t \in[\tau, \tau+\eta]$ e levando em conta que $x(\tau)=y(\tau)$, obtemos

$$
\begin{aligned}
|x(t)-y(t)| & =\left|x(\tau)+\int_{\tau}^{t} f\left(s, x_{s}\right) d s-y(\tau)-\int_{\tau}^{t} f\left(s, y_{s}\right) d s\right| \\
& =\left|\int_{\tau}^{t}\left[f\left(s, x_{s}\right)-f\left(s, y_{s}\right)\right] d s\right| .
\end{aligned}
$$


Assim, pela hipótese $(H)$, temos

$$
\begin{aligned}
|x(t)-y(t)| & \leq \int_{\tau}^{t}\left|f\left(s, x_{s}\right)-f\left(s, y_{s}\right)\right| d s \leq L \int_{\tau}^{t}|x(s)-y(s)| d s \\
& \leq L(t-\tau) \sup _{\tau \leq s \leq t}|x(s)-y(s)| \\
& \leq L \eta \sup _{\tau \leq s \leq \tau+\eta}|x(s)-y(s)| .
\end{aligned}
$$

Como tomamos $t \in[\tau, \tau+\eta]$ arbitrário, a expressão (2.51) implica

$$
(1-L \eta) \sup _{\tau \leq s \leq \tau+\eta}|x(s)-y(s)| \leq 0
$$

Daí, uma vez que podemos supor, sem perda de generalidade, que $\eta<1 / L$, então, da expressão obtida em (2.52), obtemos

$$
x(s)=y(s), \quad \tau \leq s \leq \tau+\eta
$$

e, portanto, $\tau+\eta \in A$. Mas isso é um absurdo, pois $\tau=\sup A$. Portanto $T \in A$, ou seja, $x(t)=y(t)$, para todo $t \in[0, T]$, o que encerra a demonstração. 


\section{Existência e unicidade de solução periódica para}

\section{EDFR's impulsivas neutras}

\subsection{Introdução}

Neste capítulo, nosso objeto de estudo será uma classe de equações diferenciais funcionais neutras com efeitos impulsivos. Diferentemente das condições impulsivas presentes no Capítulo 2, que eram

$$
\left.x\left(t_{k}^{+}\right)-x\left(t_{k}\right)=I\left(t_{k}, x\left(t_{k}\right)\right), \quad k \in \mathbb{N}, I \in C([0,+\infty) \times \mathbb{R}, \mathbb{R})\right),
$$

aqui, as condições impulsivas do problema que estudamos são da forma

$$
x\left(t_{k}^{+}\right)-x\left(t_{k}\right)=b_{k} x\left(t_{k}\right), \quad k \in \mathbb{N}, b_{k} \in \mathbb{R} .
$$

Os momentos de impulso do problema que estudaremos constituem uma sequência $\left\{t_{k}\right\}_{k \in \mathbb{N}}$, satisfazendo

$$
0<t_{1}<t_{2}<\ldots<t_{k}<t_{k+1}<\ldots \quad \text { e } \quad \lim _{k \rightarrow+\infty} t_{k}=+\infty
$$

Suporemos que existe uma constante $T>0$ tal que $\left\{t_{k} ; k \in \mathbb{N}\right\} \cap[0, T]=\left\{t_{1}, \ldots t_{m}\right\}$ e de modo que, para cada $k \in \mathbb{N}$, se tenha $t_{m+k}=t_{k}+T$. Suporemos dada uma sequência $\left\{b_{k}\right\}_{k \in \mathbb{N}}$ 
de números reais maiores que -1 . Denotando $\left\{b_{j_{1}}<b_{j_{2}} \ldots<b_{j_{n}}\right\}=[0, T] \cap\left\{b_{k} ; k \in \mathbb{N}\right\}$, suporemos que $b_{j_{n}+k}=b_{j_{k}}+T, k \in \mathbb{N}$. Suporemos ainda que a distância entre a origem e o maior termo negativo da sequência $\left\{b_{k}\right\}$ é igual a $T-b_{j_{n}}$, que a distância entre a origem e o segundo maior termo negativo da sequência $\left\{b_{k}\right\}$ é igual a $T-b_{j_{n-1}}$ e assim sucessivamente. Dessa forma, ppor simplicidade, poderemos denotar, sem perda de generalidade, $b_{k}=b_{j_{k}}, k=1, \ldots, n$.

Sejam $r$ e $\delta$ duas constantes tais que $0<r \leq T, 0 \leq \delta \leq r$ e $b_{j_{n}}<T-\delta$.

Nosso objetivo é encontrar condições que garantam existência e unicidade de soluções $T$ periódicas para o problema

$$
\begin{cases}x^{\prime}(t)+B x^{\prime}(t-\delta)=f\left(t, x_{t}\right), & t \geq 0, t \neq t_{k}, k \in \mathbb{N} \\ \Delta x\left(t_{k}\right)=b_{k} x\left(t_{k}\right), & k \in \mathbb{N} .\end{cases}
$$

Neste problema, $B$ é uma constante diferente de zero e a função $f:[0,+\infty) \times G([-r, 0], \mathbb{R}) \longrightarrow$ $\mathbb{R}$ é $T$-periódica na primeira variável. Por fim, lembramos que $\Delta x\left(t_{k}\right)=x\left(t_{k}^{+}\right)-x\left(t_{k}\right)$, sendo $x\left(t_{k}^{+}\right)=\lim _{t \rightarrow t_{k}^{+}} x(t)$.

Definição 3.1. Diremos que uma função $x \in G([-r,+\infty), \mathbb{R})$ é uma solução do problema (3.1), quando

(i) $x$ for absolutamente contínua em cada intervalo $\left[-\delta, t_{1}\right] e\left(t_{k}, t_{k+1}\right], k \in \mathbb{N}$;

(ii) $x$ satisfizer a igualdade $x^{\prime}(t)+B x^{\prime}(t-\delta)=f\left(t, x_{t}\right)$ em quase todo ponto de $[0,+\infty) \backslash$ $\left\{t_{k} ; k \in \mathbb{N}\right\}$

(iii) Para cada $k \in \mathbb{N}$, valer $\Delta x\left(t_{k}\right)=b_{k} x\left(t_{k}\right)$.

Diremos que uma solução x de (3.1) é T-periódica quando sua restrição a $[-\delta,+\infty)$ for uma função T-periódica. 
Neste capítulo, apresentaremos uma abordagem alternativa ao método aplicado ao problema do capítulo anterior. O procedimento que adotaremos consiste em utilizar uma equação não impulsiva auxiliar, de modo a contornar as dificuldades inerentes à existência dos momentos impulsivos $t_{k}$.

Com o intuito de utilizar uma equação não impulsiva auxiliar, fez-se necessário flexibilizar a definição de solução, em relação à definição para solução dada no capítulo anterior. De fato, enquanto no Capítulo 2 havíamos exigido que uma solução do problema (2.1) fosse diferenciável em cada intervalo $\left[0, t_{1}\right],\left(t_{k}, t_{k+1}\right], k \in \mathbb{N}$, no presente capítulo, exigiremos apenas que a solução do problema que estudamos seja absolutamente contínua nesses intervalos. No entanto, isso não enfraquecerá o resultado obtido, pois, a exemplo do que ocorreu no capítulo anterior, neste também obteremos condições que garantem a existência de solução periódica continuamente diferenciável nos intervalos $\left[0, t_{1}\right],\left(t_{k}, t_{k+1}\right], k \in \mathbb{N}$.

O procedimento adotado neste capítulo, foi inspirado no trabalho realizado por Meili Li et al em [27]. Nesse trabalho, os autores encontraram condições suficientes para a existência de soluções periódicas para a seguinte equação diferencial com retardo constante $s \leq 0$ :

$$
x^{\prime}(t)=g(t, x(t+s)), t \geq 0, t \neq t_{k}
$$

sendo $g: \mathbb{R} \times \mathbb{R} \longrightarrow \mathbb{R}$ uma função dada e a equação (3.2) sujeita à condição impulsiva

$$
x\left(t_{k}^{+}\right)-x\left(t_{k}\right)=b_{k} x\left(t_{k}\right), \quad k \in \mathbb{N},
$$

sendo $b_{k}$ um número real maior que -1 . As soluções de (3.2), (3.3) foram definidas como funções reais definidas em $\mathbb{R}$, absolutamente contínuas nos intervalos $\left[0, t_{1}\right],\left(t_{k}, t_{k+1}\right], k \in \mathbb{N}$, que satisfazem (3.2) em quase todo ponto de $[0,+\infty) \backslash\left\{t_{k} ; k \in \mathbb{N}\right\}$ e satisfazem (3.3) para cada $k \in \mathbb{N}$. 
Visando investigar a existência de solução periódica para o problema (3.2), (3.3), os autores em [27] introduziram a equação auxiliar não impulsiva

$$
u^{\prime}(t)=h(t, u(t+s), \quad t \geq 0
$$

na qual

$$
h(t, u(t+s))=\prod_{t_{k}<t}\left(1+b_{k}\right)^{-1} f\left(t, \prod_{t_{k}<t+s}\left(1+b_{k}\right) u(t+s)\right),
$$

sendo o produto acima igual a 1 quando o número de fatores for zero e $s$ o retardo constante mencionado acima.

Em seguida, os autores definiram solução para a equação (3.4) como sendo uma função absolutamente contínua definida em $\mathbb{R}$ e satisfazendo a igualdade (3.4) em quase todo ponto da semirreta não negativa. Na sequência, os autores demonstraram que a existência de solução $T$-periódica para a equação (3.4) implica o mesmo para o problema (3.2), (3.3) (ver [27], Lema 2.1).

Segundo o nosso entendimento, o estudo da equação não impulsiva (3.4) em [27] apresenta alguns problemas, que passamos a apontar. Na demonstração do resultado que apresenta condições suficientes para existência de solução $T$-periódica para a equação (3.4) (Lema 2.4, em [27]), os autores consideram o espaço $X$ das funções reais, absolutamente contínuas e $T$ periódicas, definidas em $\mathbb{R}$, munido da norma do supremo. Na sequência, definem os operadores $L: X \longrightarrow X$ pondo $L u(t)=u^{\prime}(t)$ e $N: X \longrightarrow X, N u(t)=h(t, u(t+s))$ e identificam a equação (3.4) com a equação a operadores

$$
L u=N u, \quad u \in X
$$

Feito isso, o objetivo passa a ser encontrar condições que garantam a existência de solução para a equação (3.6), mediante o uso do Teorema de Continuação de Mawhin, que já utilizamos no 
capítulo anterior e relembramos agora.

Teorema 3.2. Sejam $X, Y$ dois espaços de Banach e $W$ um subespaço de $X$. Suponha que $L: W \longrightarrow Y$ seja um operador de Fredholm de índice zero e $N: X \longrightarrow Y$ seja um operador L-compacto em $\bar{\Omega}$, sendo $\Omega$ um subconjunto aberto e limitado de X. Suponha, também, que as seguintes condições sejam satisfeitas:

(i) Se $x \in \partial \Omega \cap W$, então $L x \neq \lambda N x, \lambda \in(0,1)$;

(ii) Se $x \in \partial \Omega \cap \operatorname{Ker} L$, então $Q N x \neq 0$;

(iii) $\operatorname{deg}\{J Q N, \Omega \cap \operatorname{Ker} L, 0\} \neq 0$, sendo J $: \operatorname{Im} Q \longrightarrow$ Ker L um isomorfismo.

Nestas condições, a equação $L x=N x$ possuirá pelo menos uma solução em $\bar{\Omega} \cap W$.

Lembramos que no enunciado desse teorema, $\bar{\Omega}$ denota o fecho de $\Omega$ em $X$ e $\partial \Omega$ denota a fronteira de $\Omega$.

Neste momento, gostaríamos de chamar a atenção para dois fatos. Em primeiro lugar, da expressão que define $N$ e da definição da função $h$, dada em (3.5), podemos concluir que, diferentemente do que se afirma em [27], $N u \notin X$. De fato, $N u$ não é uma função contínua nos pontos $t_{k}$, já que a função $t \longmapsto \prod_{t_{k}<t}\left(1+b_{k}\right)$ é descontínua em tais pontos. Além disso, como a derivada de uma função absolutamente contínua não é necessariamente absolutamente contínua, então o contradomínio do operador $L$ é, em geral, um espaço que contém propriamente $X$.

Apesar dos problemas mencionadas acima, o artigo [27] nos serviu como fonte de ideias para o método de utilizar uma equação auxiliar não impulsiva no estudo da questão da existência e unicidade de solução periódica para o problema (3.1).

Por fim, cabe ressaltar que, embora em [27] não se tenha obtido condições suficientes para a unicidade de solução periódica para o problema (3.2), (3.3), os autores mostraram, com o Lema 
2.1, que é possível utilizar a equação auxiliar não impulsiva (3.4) para tratar da unicidade de solução para tal problema.

Na próxima seção, apresentaremos uma equação não impulsiva associada ao problema (3.1) e mostraremos que a existência e unicidade de solução $T$-periódica para tal equação garante que o problema (3.1) goza da mesma propriedade.

\subsection{Uma equação não impulsiva auxiliar}

Como mencionamos acima, nossa estratégia para estudar a questão da existência e unicidade de solução $T$-periódica para o problema impulsivo (3.1) será investigar a mesma questão relacionada a uma equação auxiliar não impulsiva. Tal equação estará de tal maneira associada ao problema impulsivo (3.1) que a existência e unicidade de solução $T$-periódica para essa equação garantirá a mesma propriedade para o problema impulsivo.

Considere a equação

$$
u^{\prime}(t)+B \beta(t)^{-1} \beta(t-\delta) u^{\prime}(t-\delta)=h\left(t, u_{t}\right), \quad t \geq 0,
$$

sendo $h:[0,+\infty) \times G([-r, 0], \mathbb{R}) \longrightarrow \mathbb{R}$ dada por

$$
h(t, \varphi)=\frac{f\left(t, \beta_{t} \varphi\right)}{\beta(t)}
$$

e $\beta:[-r,+\infty) \longrightarrow(0,+\infty)$, dada por $\beta(t)=\prod_{t_{k}<t}\left(1+b_{k}\right)$ se $t>t_{1}$ e $\beta(t)=\prod_{k=1}^{n}\left(1+b_{k}\right)$ se $t \in\left[-r, t_{1}\right]$.

Como a função $h$ depende exclusivamente de $f$ e $\beta$, que provêm do problema impulsivo (3.1), vemos que a equação (3.7) está diretamente associada ao problema impulsivo (3.1).

Definição 3.3. Diremos que $u \in G([-r,+\infty), \mathbb{R})$ é uma solução da equação (3.7), quando as 
condições seguintes forem satisfeitas:

(i) u for absolutamente contínua em $[-\delta,+\infty)$;

(ii) u satisfizer a igualdade $u^{\prime}(t)+B \beta(t)^{-1} \beta(t-\delta) u^{\prime}(t-\delta)=h\left(t, u_{t}\right)$ em quase todo ponto $t \in[0,+\infty)$.

O próximo resultado nos diz que o modo como a equação (3.7) está associada ao problema impulsivo (3.1) é tal que da existência e unicidade de solução $T$-periódica para a equação podemos inferir a mesma propriedade para o problema impulsivo.

Lema 3.4. Se $u \in G([-r,+\infty), \mathbb{R})$ for solução da equação (3.7), então a função $x \in G([-r,+\infty), \mathbb{R})$ dada por $x(t)=\beta(t) u(t)$ será solução do problema (3.1). Reciprocamente, se $x \in G([-r,+\infty), \mathbb{R})$ for solução do problema (3.1), então a função $u \in G([-r,+\infty), \mathbb{R})$ dada por $u(t)=x(t) / \beta(t)$ será solução da equação (3.7).

Demonstração. Suponha que $u \in G([-r,+\infty), \mathbb{R})$ seja solução da equação (3.7). Considere a função $x \in G([-r,+\infty), \mathbb{R})$ dada por $x(t)=\beta(t) u(t)$. Na sequência, provaremos que a função $x$ cumpre as condições dadas na Definição 3.1 de solução do problema (3.1).

Como $u$ e $\beta$ são absolutamente contínuas em cada intervalo $\left[-\delta, t_{1}\right],\left(t_{k}, t_{k+1}\right], k \in \mathbb{N}$, o mesmo podemos dizer da função $x$, ou seja, $x$ cumpre a primeira condição dada na Definição 3.1.

Por outro lado, como $u$ é solução da equação (3.7), então, pelo item (ii) da Definição 3.3, sabemos que, para quase todo $t \in[0,+\infty) \backslash\left\{t_{k} ; k \in \mathbb{N}\right\}$, vale

$$
\begin{gathered}
x^{\prime}(t)+B x^{\prime}(t-\delta)-f\left(t, x_{t}\right)= \\
=\beta(t) u^{\prime}(t)+B \beta(t-\delta) u^{\prime}(t-\delta)-f\left(t, \beta_{t} u_{t}\right)=
\end{gathered}
$$




$$
\begin{aligned}
& =\beta(t)\left[u^{\prime}(t)+B \beta(t)^{-1} \beta(t-\delta) u^{\prime}(t-\delta)-\frac{f\left(t, \beta_{t} u_{t}\right)}{\beta(t)}\right]= \\
& =\beta(t)\left[u^{\prime}(t)+B \beta(t)^{-1} \beta(t-\delta) u^{\prime}(t-\delta)-h\left(t, u_{t}\right)\right]=0,
\end{aligned}
$$

mostrando que $x$ satisfaz a condição $(i i)$ da Definição 3.1 .

Finalmente, vejamos que a função $x$ satisfaz a condição (iii) da referida definição, ou seja, vejamos que $\Delta x\left(t_{k}\right)=b_{k} x\left(t_{k}\right), k \in \mathbb{N}$. De fato, pois para cada $k \in \mathbb{N}$, temos

$$
\Delta x\left(t_{k}\right)=\lim _{t \rightarrow t_{k}^{+}} x(t)-x\left(t_{k}\right)=\lim _{t \rightarrow t_{k}^{+}} u(t) \beta(t)-x\left(t_{k}\right)=\lim _{t \rightarrow t_{k}^{+}} u(t) \prod_{t_{j}<t}\left(1+b_{j}\right)-x\left(t_{k}\right),
$$

ou seja,

$$
\begin{aligned}
\Delta x\left(t_{k}\right) & =u\left(t_{k}\right) \prod_{t_{j} \leq t_{k}}\left(1+b_{j}\right)-x\left(t_{k}\right)=\frac{x\left(t_{k}\right)}{\beta\left(t_{k}\right)}\left(1+b_{k}\right) \prod_{t_{j}<t_{k}}\left(1+b_{j}\right)-x\left(t_{k}\right) \\
& =\frac{x\left(t_{k}\right)}{\beta\left(t_{k}\right)}\left(1+b_{k}\right) \beta\left(t_{k}\right)-x\left(t_{k}\right)=b_{k} x\left(t_{k}\right) .
\end{aligned}
$$

Reciprocamente, suponha que $x \in G([-r,+\infty), \mathbb{R})$ seja uma solução do problema (3.1) e considere a função $u \in G([-r,+\infty), \mathbb{R})$ definida por $u(t)=x(t) / \beta(t)$.

Como $x$ e $\beta$ são absolutamente contínuas em cada intervalo $\left[-\delta, t_{1}\right],\left(t_{k}, t_{k+1}\right], k \in \mathbb{N}$, então a função $u$ também o é. Para concluir que $u$ cumpre a condição $(i)$ da Definição 3.3, isto é, que $u$ é absolutamente contínua em $[0,+\infty)$, resta mostrar que ela é contínua pela direita em cada ponto $t_{k}$. De fato isso ocorre, pois para cada $k \in \mathbb{N}$, temos

$$
\begin{aligned}
\lim _{t \rightarrow t_{k}^{+}} u(t) & =\lim _{t \rightarrow t_{k}} x(t) / \beta(t)=\lim _{t \rightarrow t_{k}^{+}} x(t) \prod_{t_{j}<t}\left(1+b_{j}\right)^{-1}=x\left(t_{k}^{+}\right) \prod_{t_{j} \leq t_{k}}\left(1+b_{j}\right)^{-1} \\
& =\left(1+b_{k}\right) x\left(t_{k}\right)\left(1+b_{k}\right)^{-1} \prod_{t_{j}<t}\left(1+b_{j}\right)^{-1}=x\left(t_{k}\right) / \beta\left(t_{k}\right)=u\left(t_{k}\right) .
\end{aligned}
$$


Por fim, vejamos que a função $u$ cumpre a condição (ii) da Definição 3.3, ou seja, que $u$ satisfaz a igualdade $u^{\prime}(t)=h\left(t, u_{t}\right)$ em quase todo ponto de $[0,+\infty)$. Como $x$ é solução do problema (3.1), então, para quase todo $t \in[0,+\infty)$, vale

$$
\begin{aligned}
u^{\prime}(t) & =x^{\prime}(t) / \beta(t)=\left[f\left(t, x_{t}\right)-B x^{\prime}(t-\delta)\right] / \beta(t) \\
& =\left[f\left(t, \beta_{t} u_{t}\right)-B \beta(t-\delta) u^{\prime}(t-\delta)\right] / \beta(t),
\end{aligned}
$$

ou seja

$$
u^{\prime}(t)=h\left(t, u_{t}\right)-B \beta(t)^{-1} \beta(t-\delta) u^{\prime}(t-\delta),
$$

e o resultado está demonstrado.

Como o Lema 3.4 nos diz que a existência e unicidade de solução para a equação não impulsiva (3.7) implicam na existência e unicidade de solução para o problema impulsivo (3.1), então, para contornar as dificuldades decorrentes da existência dos impulsos presentes nesse problema, nos concentraremos, nas duas próximas seções, em obter condições que garantam a existência e unicidade de solução $T$-periódica para a equação (3.7).

\subsection{Existência de solução periódica}

Considere as seguintes condições:

$(H 1)$ Se $\varphi:[-r,+\infty) \longrightarrow \mathbb{R}$ for tal que $\varphi_{t} \in G([-r, 0], \mathbb{R})$ para cada $t \geq 0$, então a função $t \mapsto f\left(t, \varphi_{t}\right)$, definida para $t \geq 0$, será contínua;

(H2) Existe uma constante $d>0$ tal que se $\varphi \in G([-r, 0], \mathbb{R})$ e $|\varphi(0)| \geq d$, então

$$
\varphi(0)\left[f\left(t, \beta_{t} \varphi\right)\right]>0, \quad t \in[0, T]
$$


(H3)

$$
B<l / L, \text { sendo } l=\min _{-r \leq t \leq T} \beta(t) \text { e } L=\max _{-r \leq t \leq T} \beta(t)
$$

(H4) Existe uma constante positiva $b<\left(1-B L l^{-1}\right) / T$ tal que se $\varphi, \psi \in G([-r, 0], \mathbb{R})$, então

$$
|f(t, \varphi)-f(t, \psi)| \leq b|\varphi(t)-\psi(t)|, \quad t \in[0, T]
$$

Nosso objetivo na sequência, será demonstrar o resultado seguinte.

Teorema 3.5. Se as condições (H1) a (H4) forem cumpridas, então a equação (3.7) possuirá pelo menos uma solução $T$-periódica e continuamente diferenciável nos intervalos $\left[0, t_{1}\right],\left(t_{k}, t_{k+1}\right]$, $k \in \mathbb{N}$.

A demonstração deste teorema será apresentada ao final desta seção e seguirá da série de resultados que obteremos a partir de agora. Mostraremos que o Teorema 3.5 é uma consequência do Teorema 3.2. Para tanto, nossa estratégia será encontrar uma equação a operadores $L x=N x$, relacionada de tal modo à equação (3.7) que da existência de solução para tal equação poderemos inferir a existência de solução para a equação (3.7) com as propriedades enunciadas no Teorema 3.5.

Sejam $X=G([0, T], \mathbb{R})$ e $W$ o subespaço de $X$ formado pelas funções $x \in X$ absolutamente contínuas, de classe $C^{1}$ nos intervalos $\left[0, t_{1}\right],\left(t_{1}, t_{2}\right], \ldots,\left(t_{m}, T\right]$, satisfazendo $x(0)=x(T)$ e tais que os limites laterais $x^{\prime}\left(t_{k}^{+}\right)$existem e são finitos.

Defina o operador $A: W \longrightarrow X$ por

$$
A x(t)=x(t)+B \beta(t)^{-1} \beta(t-\delta) x(t-\delta), \quad t \geq 0
$$

e os operadores $L: W \longrightarrow X$ e $N: X \longrightarrow X$ pondo

$$
L x(t)=A x^{\prime}(t) \quad \text { e } \quad N x(t)=h\left(t, x_{t}\right), \quad t \geq 0 .
$$


Com respeito à definição de $L$, convém observar que, nos pontos $t_{k} \in[0, T], x^{\prime}\left(t_{k}\right)$ denota a derivada de $x$ à esquerda de $t_{k}$. Além disso, nas definições dos operadores $A$ e $N$, consideramos que $x(t)=x(t+T)$ para cada $t \in[-r, 0]$.

Das definições dadas acima, podemos concluir que se a equação a operadores

$$
L x=N x
$$

possuir solução, então a equação (3.7) possuirá solução com as propriedades enunciadas no Teorema 3.5. De fato, suponha que a equação (3.11) possua uma solução $y \in W$. Neste caso, a função $x \in G([-r,+\infty), \mathbb{R})$ dada por $x(t)=y(t)$ se $t \in[0, T], x(t)=x(t+T)$ se $t \in[-r, 0]$ e $x(t)=x(t-n T)$ se $t \in(n T,(n+1) T], n \in \mathbb{N}$, será $T$-periódica, será de classe $C^{1}$ nos intervalos $\left[0, t_{1}\right],\left(t_{k}, t_{k+1}\right], k \in \mathbb{N}$ e, pela Definição $3.3, x$ será uma solução da equação (3.7).

Dessa forma, com o intuito de demonstrar o Teorema 3.5, mostraremos que se as condições (H1) a (H4) forem satisfeitas, então a equação (3.11) possuirá pelo menos uma solução. Para tanto, mostraremos que as condições $(H 1)$ a $(H 4)$ implicam que as hipóteses do Teorema 3.2 são satisfeitas, com $L$ e $N$ definidos como acima.

Iniciamos provando que o operador linear $L$ é um operador de Fredholm de índice zero. Para tanto, o próximo resultado será importante.

Lema 3.6. Sejam $E$ um espaço de Banach e $T: E \longrightarrow E$ um operador linear limitado tal que $\|T\|<1$. Então $(I-T)$ é um operador bijetivo e $(I-T)^{-1}$ é limitado e

$$
\left\|(I-T)^{-1}\right\| \leq \frac{1}{1-\|T\|}
$$

O resultado a seguir é uma consequência do lema acima e será útil na demonstração de que o operador $L$ é de Fredholm de índice zero. 
Lema 3.7. Se a condição (H3) for satisfeita, então o operador A, definido em (3.9), será bijetivo $e\left\|A^{-1}\right\| \leq l /(l-B L)$.

Demonstração. Considere o operador $T: X \longrightarrow X$ dado por

$$
T x(t)=-B \beta(t)^{-1} \beta(t-\delta) x(t-\delta) .
$$

Como para cada $x \in X$, vale $x(t)=x(t+T), t \in[-\delta, 0]$, então

$$
\begin{aligned}
\|T x\|_{\infty} & =\sup _{t \in[0, T]} B \beta(t)^{-1} \beta(t-\delta)|x(t-\delta)| \\
& \leq \frac{B L}{l} \sup _{t \in[0, T]}|x(t-\delta)|=\frac{B L}{l} \sup _{t \in[0, T]}|x(t)|=\frac{B L}{l}\|x\|_{\infty},
\end{aligned}
$$

qualquer que seja $x \in X$. Dessa forma, se a condição $(H 3)$ for satisfeita, teremos

$$
\|T\| \leq \frac{B L}{l}<1
$$

Então, pelo Lema 3.6, o operador $A=\left.(I-T)\right|_{W}$ será bijetivo e

$$
\left\|A^{-1}\right\|=\left\|\left.(I-T)\right|_{W} ^{-1}\right\| \leq\left\|I-\left.T\right|^{-1}\right\| \leq \frac{1}{1-\|T\|}
$$

Disto e de (3.12), obteremos

$$
\left\|A^{-1}\right\| \leq \frac{l}{l-B L},
$$

e o resultado está provado.

Estamos em condições agora de provar o resultado seguinte.

Lema 3.8. Se a condição (H3) for satisfeita, então o operador $L: W \longrightarrow X$, definido em (3.10), 
será de Fredholm de índice zero.

Demonstração. Suponha que se cumpre a condição (H3). Seja $y \in X$ tal que $y=L x$, para algum $x \in W$. Então

$$
\begin{aligned}
\int_{0}^{T} y(t) d t & =\int_{0}^{T} L x(t) d t=\int_{0}^{T} A x^{\prime}(t) d t=\int_{0}^{T}\left[x^{\prime}(t)+B \beta(t)^{-1} \beta(t-\delta) x^{\prime}(t-\delta)\right] d t \\
& =\int_{0}^{T} x^{\prime}(t) d t+B \int_{0}^{T} \beta(t)^{-1} \beta(t-\delta) x^{\prime}(t-\delta) d t \\
& =[x(T)-x(0)]+B\left[\beta(T)^{-1} \beta(T-\delta) x(T-\delta)-\beta(0)^{-1} \beta(-\delta) x(-\delta)\right] \\
& =0 .
\end{aligned}
$$

Fica assim provado que

$$
\operatorname{Im} L \subset\left\{y \in X ; \int_{0}^{T} y(t) d t=0\right\}
$$

Reciprocamente, dado $y \in X$ tal que $\int_{0}^{T} y(t) d t=0$, considere a função a função $x:[0, T] \longrightarrow$ $\mathbb{R}, x(t)=A^{-1}\left(\int_{0}^{t} y(s) d s\right)$. Então

$$
L x(t)=A x^{\prime}(t)=\frac{d}{d t}[A x(t)]=\frac{d}{d t}\left[\int_{0}^{t} y(s) d s\right]=y(t)
$$

o que, juntamente com (3.13), nos permite concluir que

$$
\operatorname{Im} L=\left\{y \in X ; \int_{0}^{T} y(t) d t=0\right\}
$$

Com a mesma prova do Lema 2.3, do capítulo anterior, mostra-se que $\operatorname{Im} L$ é fechado em $G([0, T], \mathbb{R})$. Para concluir que $L$ é um operador de Fredholm de índice zero, resta mostrar que 
$\operatorname{dim} \operatorname{Ker} L=$ codim $\operatorname{Im} L<+\infty$. Provaremos que $\operatorname{dim} \operatorname{Ker} L=\operatorname{codim} \operatorname{Im} L=1$.

Vejamos que $\operatorname{Ker} L$ pode ser identificado com $\mathbb{R}$. Se $x \in W$ for uma função constante, então $x^{\prime}(t)=0$ para cada $t \in[0, T]$. Daí, como $x(t)=x(t+T)$ para cada $t \in[-\delta, 0]$, então $x$ também é constante em $[-\delta, 0]$ e, portanto, $x^{\prime}(t)=0$ para cada $t \in[-\delta, 0]$. Consequentemente,

$$
L x(t)=A x^{\prime}(t)=x^{\prime}(t)+B \beta(t)^{-1} \beta(t-\delta) x^{\prime}(t-\delta)=0, \quad t \in[0, T] .
$$

Reciprocamente, se $x \in \operatorname{Ker} L$, então $0=L x=A x^{\prime}$ e, por conseguinte, $x^{\prime}=A^{-1} 0=0$. Assim, se $x \in \operatorname{Ker} L$, então $x$ é uma função constante. Dessa forma, mostramos que $\operatorname{dim} \operatorname{Ker} L=1$.

Para finalizar a demonstração, resta mostrar que codim $\operatorname{Im} L=1$. Defina o operador linear

$$
Q x:=\frac{1}{T} \int_{0}^{T} x(t) d t, \quad x \in X
$$

Então

$$
Q(Q x(t))=\frac{1}{T} \int_{0}^{T} Q x(t) d t=\frac{1}{T} \int_{0}^{T}\left[\frac{1}{T} \int_{0}^{T} x(\tau) d \tau\right] d t=\frac{1}{T} \int_{0}^{T} x(t) d t=Q x(t)
$$

mostrando que $Q$ é um operador idempotente. Além disso, $\operatorname{Im} Q \cap \operatorname{Ker} Q=\{0\}$. De fato, se $y \in \operatorname{Im} Q$, então para algum $x \in W$, temos

$$
y=\frac{1}{T} \int_{0}^{T} x(t) d t
$$

ou seja, $y$ é constante. Por outro lado, se $y$ for constante e $y \in \operatorname{Ker} Q$, então, claramente, $y=0$. Assim, além de $Q$ ser linear e idempotente, temos $\operatorname{Im} Q \cap \operatorname{Ker} Q=\{0\}$. Dessa, podemos escrever $X=\operatorname{Ker} Q \oplus \operatorname{Im} Q$. Como, por outro lado, $\operatorname{Im} Q \simeq \mathbb{R}$, então codim $\operatorname{Ker} Q=1$. Daí, como, por (3.14), Im $L=\operatorname{Ker} Q$, concluímos que codim $\operatorname{Im} L=1$ e o resultado está demonstrado. 
Nosso objetivo, na sequência, será encontrar um aberto e limitado $\Omega$ em $X$, de modo que $N$ seja $L$-compacto no fecho de $\Omega$, que denotamos por $\bar{\Omega}$, e de modo que se cumpram as condições (i), (ii) e (iii) do Teorema 3.2. Encontraremos o conjunto $\Omega$ a partir de uma estimativa a priori para soluções da equação

$$
L x(t)=\lambda N x(t), \quad \lambda \in(0,1) .
$$

Mais precisamente, mostraremos que existe uma constante positiva $D$, independente de $\lambda$, que majora todas as possíveis soluções da equação (3.16). Uma vez obtida tal constante $D$, tomaremos como aberto limitado $\Omega \subset X$, o subespaço de $X$ constituído pelas funções pertencentes a $X$, com norma menor que $D$.

Com os dois próximos resultados, obtemos a estimativa a priori para soluções da equação (3.16).

Lema 3.9. Suponha que as condições (H1) a (H4) sejam satisfeitas. Se $x \in W$ for uma solução da equação (3.16), então

$$
\|x\|_{\infty}<d+\sqrt{T}\left\|x^{\prime}\right\|_{2}
$$

sendo $\left\|x^{\prime}\right\|_{2}=\left(\int_{0}^{T} x^{\prime}(t)^{2} d t\right)^{\frac{1}{2}}$

Demonstração. Suponha que a equação (3.16) possua uma solução $x \in W$. Integrando de 0 a $T$ ambos os membros dessa equação, obtemos

$$
\int_{0}^{T}\left[x^{\prime}(t)+B \beta(t)^{-1} \beta(t-\delta) x^{\prime}(t-\delta)\right] d t=\lambda \int_{0}^{T} h\left(t, x_{t}\right) d t
$$

Daí, como $x(0)=x(T), x(T-\delta)=x(-\delta)$ e como, pela hipótese $(H 3), \beta(T)^{-1} \beta(T-\delta)=$ $\beta(0)^{-1} \beta(-\delta)$, então, usando integração por partes, concluímos que o membro esquerdo da 
igualdade (3.17) é igual a zero. Por conseguinte, temos

$$
\int_{0}^{T} h\left(t, x_{t}\right) d t=0
$$

ou seja, pela definição de $h$, dada em (3.8), temos

$$
\int_{0}^{T} \beta(t)^{-1}\left[f\left(t, \beta_{t} x_{t}\right)\right] d t=0
$$

Daí, como $\beta$ é uma função positiva e, pelas hipóteses $(H 1)$ e $(H 2)$, a função

$$
t \mapsto f\left(t, \beta_{t} x_{t}\right), \quad t \in[0, T]
$$

é contínua, então existe $\tau \in[0, T]$ tal que

$$
f\left(\tau, \beta_{\tau} x_{\tau}\right)=0
$$

Isso implica $|x(\tau)|<d$. De fato, se tivéssemos $|x(\tau)| \geq d$, então $\left|x_{\tau}(0)\right| \geq d$ o que, pela hipótese (H4), implicaria

$$
x_{\tau}(0) f\left(\tau, \beta_{\tau} x_{\tau}\right)>0
$$

que está em contradição com (3.19). Portanto $|x(\tau)|<d$. Disso, da desigualdade triangular e da desigualdade de Hölder, obtemos

$$
|x(t)|=\left|x(\tau)+\int_{\tau}^{t} x^{\prime}(s) d s\right|<d+\int_{0}^{T}\left|x^{\prime}(s)\right| d s \leq d+\sqrt{T}\left\|x^{\prime}\right\|_{2}, \quad t \in[0, T]
$$

o que completa a demonstração.

Com o próximo resultado, completamos a lista de condições suficientes para a obtenção de uma estimativa a priori para soluções de (3.16). 
Lema 3.10. Se as condições (H1) a (H4) forem satisfeitas, então existirá uma constante $D>0$, independente de $\lambda$, tal que se $x \in W$ for solução da equação (3.16), então $\|x\|_{\infty}<D$.

Demonstração. Suponha que se cumprem as condições $(H 1)$ a $(H 4)$ e suponha que a equação (3.16) possua uma solução $x \in W$. Multiplicando ambos os membros da equação (3.16) por $x^{\prime}(t)$, obtemos

$$
\left[x^{\prime}(t)\right]^{2}+B \beta(t)^{-1} \beta(t-\delta) x^{\prime}(t) x^{\prime}(t-\delta)=\lambda h\left(t, x_{t}\right) x^{\prime}(t)
$$

Então, usando a definição de $h$, a desigualdade triangular e o fato de que $l \leq \beta(t) \leq L$ para todo $-r \leq t \leq T$, obtemos

$$
\begin{aligned}
{\left[x^{\prime}(t)\right]^{2} } & <\beta(t)^{-1}\left|f\left(t, \beta_{t} x_{t}\right)\right|\left|x^{\prime}(t)\right|+\beta(t)^{-1}\left|x^{\prime}(t)\right|+B L l^{-1}\left|x^{\prime}(t)\right|\left|x^{\prime}(t-\delta)\right| \\
& \leq \beta(t)^{-1}\left|f\left(t, \beta_{t} x_{t}\right)-f(0,0)\right|\left|x^{\prime}(t)\right|+l^{-1}|f(0,0)|\left|x^{\prime}(t)\right|+B L l^{-1}\left|x^{\prime}(t)\right|\left|x^{\prime}(t-\delta)\right| .
\end{aligned}
$$

Isto e a hipótese $(H 4)$ implicam

$$
\begin{aligned}
{\left[x^{\prime}(t)\right]^{2} } & \leq b|x(t)|\left|x^{\prime}(t)\right|+l^{-1}|f(0,0)|\left|x^{\prime}(t)\right|+B L l^{-1}\left|x^{\prime}(t)\right|\left|x^{\prime}(t-\delta)\right| \\
& \leq b\|x\|_{\infty}\left|x^{\prime}(t)\right|+l^{-1}|f(0,0)|\left|x^{\prime}(t)\right|+B L l^{-1}\left|x^{\prime}(t)\right|\left|x^{\prime}(t-\delta)\right| .
\end{aligned}
$$

Portanto, integrando de 0 a $T$ ambos os membros desta igualdade, podemos concluir, pela desigualdade de Hölder, que

$$
\left\|x^{\prime}\right\|_{2}^{2} \leq \sqrt{T} b\|x\|_{\infty}\left\|x^{\prime}\right\|_{2}+\sqrt{T} l^{-1}|f(0,0)|\left\|x^{\prime}\right\|_{2}+B L l^{-1}\left\|x^{\prime}\right\|_{2}^{2} .
$$


Por outro lado, pelo Lema 3.9, $\|x\|_{\infty} \leq d+\sqrt{T}\left\|x^{\prime}\right\|_{2}$. Disto e de (3.20), obtemos

$$
\left\|x^{\prime}\right\|_{2}^{2} \leq b T\left\|x^{\prime}\right\|_{2}^{2}+\left(b d+l^{-1}|f(0,0)|+B L l^{-1}\left\|x^{\prime}\right\|_{2}^{2} .\right.
$$

Portanto, como $b<\left(1-B L l^{-1}\right) / T$, temos

$$
\left\|x^{\prime}\right\|_{2} \leq \frac{\left(b d+l^{-1}|f(0,0)|\right) \sqrt{T}}{1-b T-B L l^{-1}}
$$

o que, pelo Lema 3.9, implica

$$
\|x\|_{\infty}<D:=d+\frac{\left(b d+l^{-1}|f(0,0)|\right) T}{1-b T-B L l^{-1}}
$$

finalizando a demonstração.

Como mencionamos anteriormente, vamos utilizar a estimativa a priori dada pelo Lema 3.10 para obter um aberto e limitado $\Omega \subset X$, de modo que o operador $N$ seja $L$-compacto em $\bar{\Omega}$ e de modo que sejam satisfeitas as condições $(i)$, (ii) e (iii) do Teorema 3.2.

Lema 3.11. Se as condições (H1) a (H4) forem satisfeitas, então existirá um aberto e limitado $\Omega \subset X$ tal que o operador $N$ é L-compacto em $\bar{\Omega}$.

Demonstração. Suponha que se cumpram as condições $(H 1)$ a $(H 4)$. De acordo com o Lema 3.10, existe uma constante $D>0$ que independe de $\lambda$ tal que se $x$ for solução da equação (3.16), então $\|x\|_{\infty}<D$. Fixe $M>\max \{D, d\}$, sendo $d$ a constante positiva presente na hipótese (H2), e defina o conjunto

$$
\Omega=\left\{x \in X ;\|x\|_{\infty}<M\right\} .
$$

Vamos mostrar que o operador $N$ é $L$-compacto em $\bar{\Omega}$. 
Defina os operadores $P: X \longrightarrow X$ e $Q: X \longrightarrow X$, pondo

$$
P x=x(0) \quad \text { e } \quad Q x=\frac{1}{T} \int_{0}^{T} x(t) d t
$$

Então $\operatorname{Im} P=\operatorname{Ker} L \mathrm{e}$, por (3.14), $\operatorname{Ker} Q=\operatorname{Im} L$.

Devemos mostrar que o conjunto $Q N(\bar{\Omega})$ é limitado e que o operador $L_{P}^{-1}(I-Q) N$ é compacto, sendo $L_{P}^{-1}$ a inversa da restrição de $L$ a $\operatorname{Ker} P$. Iniciamos mostrando que o conjunto $Q N(\bar{\Omega})$ é limitado.

Para cada $x \in \bar{\Omega}$, temos

$$
\|Q N x\|_{\infty}=\frac{1}{T}\left|\int_{0}^{T} N x(t) d t\right|=\frac{1}{T}\left|\int_{0}^{T} \frac{f\left(t, \beta_{t} x_{t}\right)}{\beta(t)} d t\right| \leq \frac{1}{T} \int_{0}^{T} \frac{\left|f\left(t, \beta_{t} x_{t}\right)\right|}{\beta(t)} d t .
$$

Por outro lado, pela hipótese $(H 1)$, existe $R>0$ tal que $\mid f\left(t, \beta_{t} x_{t} \mid \leq R\right.$ para todo $t \in[0, T]$. Como, além disso, $\beta(t) \leq l^{-1}$ para todo $t \in[0, T]$, então, de (3.22), obtemos

$$
\|Q N x\|_{\infty} \leq \frac{R}{l}
$$

A seguir, mostramos que o operador $L_{P}^{-1}(I-Q) N$ é compacto. Com o intuito de encontrar $L_{P}^{-1}(I-Q) N$, vamos buscar a expressão que define $L_{P}^{-1}: L(W \cap \operatorname{Ker} P) \longrightarrow W \cap \operatorname{Ker} P$. Seja $y \in L(W \cap \operatorname{Ker} P)$. Considere a função $x \in W \cap \operatorname{Ker} P$ dada por $x(t)=\int_{0}^{t} A^{-1} y(s) d s$. Então

$$
L x(t)=A x^{\prime}(t)=A A^{-1} y(t)=y(t) .
$$

Dessa forma,

$$
L_{P}^{-1} y(t)=\int_{0}^{t} A^{-1} y(s) d s, \quad t \in[0, T]
$$


Assim,

$$
L_{P}^{-1}(I-Q) N x(t)=\int_{0}^{t} A^{-1}(I-Q) N x(s) d s, \quad t \in[0, T]
$$

Sejam $\Lambda \subset \bar{\Omega}$ e $\left\{x^{n}\right\}$ uma sequência de funções em $\Lambda$. Para concluir que $L_{P}^{-1}(I-Q) N$ é compacto, vamos mostrar que existe uma subsequência $\left\{x^{n_{k}}\right\} \subset\left\{x^{n}\right\}$ tal que $\left\{L_{P}^{-1}(I-Q) N x^{n_{k}}\right\}$ é convergente. Para tanto, vamos mostrar que a sequência de funções $\left\{L_{P}^{-1}(I-Q) N x^{n}\right\}$ é equicontínua e uniformemente limitada. Deste fato e do Teorema de Arzelá-Ascoli seguirá a existência da subsequência convergente $\left\{x^{n_{k}}\right\}$.

No que segue, por simplicidade, denotaremos $F_{n}:=L_{P}^{-1}(I-Q) N x^{n}$. Começamos mostrando que a sequência $\left\{F_{n}\right\}$ é equicontínua. Fixe $t_{0} \in[0, T]$ e $\varepsilon>0$. Vamos exibir $\delta=\delta\left(t_{0}, \varepsilon\right)>0$ tal que, para cada $n \in \mathbb{N}$, tenhamos

$$
t \in[0, T],\left|t-t_{0}\right|<\delta \Longrightarrow\left|F_{n}(t)-F_{n}\left(t_{0}\right)\right|<\varepsilon
$$

De (3.23), segue-se que para cada $n \in \mathbb{N}$, vale

$$
\begin{aligned}
\left|F_{n}(t)-F_{n}\left(t_{0}\right)\right| & =\left|\int_{0}^{t} A^{-1}(I-Q) N x^{n}(s) d s-\int_{0}^{t_{0}} A^{-1}(I-Q) N x^{n}(s) d s\right| \\
& =\left|\int_{t_{0}}^{t} A^{-1}(I-Q) N x^{n}(s) d s\right| \\
& \leq \int_{t_{0}}^{t}\left|A^{-1}(I-Q) N x^{n}(s)\right| d s .
\end{aligned}
$$


Portanto,

$$
\begin{aligned}
\left|F_{n}(t)-F_{n}\left(t_{0}\right)\right| & \leq\left\|A^{-1}(I-Q) N x^{n}\right\|_{\infty}\left|t-t_{0}\right| \\
& \leq\left\|A^{-1}\right\|\left\|(I-Q) N x^{n}\right\|_{\infty}\left|t-t_{0}\right| .
\end{aligned}
$$

Portanto, como pelo Lema 3.7 temos $\left\|A^{-1}\right\| \leq l /(l-B L)$, então de 3.25, obtemos

$$
\left|F_{n}(t)-F_{n}\left(t_{0}\right)\right| \leq\left\|(I-Q) N x^{n}\right\|_{\infty} \frac{l\left|t-t_{0}\right|}{l-B L}
$$

Por outro lado, usando as definições de $N$ e $Q$, podemos concluir que, para cada $x \in X$, vale

$$
\begin{aligned}
|(I-Q) N x(t)| & =\left|\frac{f\left(t, \beta_{t} x_{t}\right)}{\beta(t)}-\frac{1}{T} \int_{0}^{T} \frac{f\left(t, \beta_{t} x_{t}\right)}{\beta(t)}\right| \\
& \leq \frac{2 R}{l}, \quad t \in[0, T] .
\end{aligned}
$$

De (3.25), (3.27), obtemos

$$
\left|F_{n}(t)-F_{n}\left(t_{0}\right)\right| \leq \frac{2 R\left|t-t_{0}\right|}{l-B L}
$$

Portanto, tomando $\delta<\varepsilon(l-B L) / 2 R$, obtemos a expressão dada em (3.24) e, dessa forma, provamos que a sequência de funções $\left\{F_{n}\right\}$ é equicontínua.

Vejamos, agora, que a sequência $\left\{F_{n}\right\}$ é uniformemente limitada. De (3.27) e do fato de que 
$\left\|A^{-1}\right\| \leq l /(l-B L)$, concluímos que, para cada $n \in \mathbb{N}$ e $t \in[0, T]$, vale

$$
\begin{aligned}
\left|F_{n}(t)\right| & =\left|\int_{0}^{t} A^{-1}(I-Q) N x^{n}(s) d s\right| \leq \int_{0}^{t}\left|A^{-1}(I-Q) N x^{n}(s)\right| d s \\
& \leq T\left\|A^{-1}\right\|\left\|(I-Q) N x^{n}\right\|_{\infty} \leq \frac{2 T R}{l-B L},
\end{aligned}
$$

mostrando que $\left\{F_{n}\right\}$ é uniformemente limitada e encerrando a prova de que $L_{P}^{-1}(I-Q) N$ é compacto.

Com o próximo resultado, encerramos a prova de que se $L$ e $N$ forem os operadores definidos em (3.10) e se forem satisfeitas as condições $(H 1)$ a $(H 4)$, então serão satisfeitas todas as hipóteses do Teorema 1.14.

Lema 3.12. Suponha que as condições $(H 1)$ a (H4) estejam satifeitas. Se $\Omega$ for o conjunto definido em (3.21), então as condições seguintes serão satisfeitas:

(i) $x \in \partial \Omega \cap W \Longrightarrow L x \neq \lambda N x, \lambda \in(0,1)$;

(ii) $x \in \partial \Omega \cap \operatorname{Ker} L \Longrightarrow Q N x \neq 0$;

(iii) $\operatorname{deg}\{J Q N, \Omega \cap \operatorname{Ker} L, 0\} \neq 0$, sendo $J: \operatorname{Im} Q \longrightarrow$ Ker L um isomorfismo.

Demonstração. Como $M>D$, então, pelo Lema 3.10, $L x \neq \lambda N x$, quaisquer que sejam $x \in$ $\partial \Omega \cap W$ e $\lambda \in(0,1)$, ou seja, a condição $(i)$ é satisfeita.

Provemos que se cumpre a condição (ii). Seja $x \in \partial \Omega \cap$ Ker L. Devemos mostrar que $Q N x \neq 0$ ou, equivalentemente, $\int_{0}^{T} N x(t) d t \neq 0$.

Como $x \in \operatorname{Ker} L$ e $\operatorname{Ker} L$ pode ser identificado com $\mathbb{R}$, então $x$ é uma função constante. Como, além disso, $x \in \partial \Omega$, então ou $x \equiv M$ ou $x \equiv-M$. Se valer o primeiro caso, então 
$x_{t}(0)=M>d, t \in[0, T]$, o que, por $(H 4)$, implica

$$
f\left(t, \beta_{t} x_{t}\right)>0, \quad t \in[0, T]
$$

Portanto,

$$
\int_{0}^{T} N x(t) d t=\int_{0}^{T} \beta(t)^{-1} f\left(t, \beta_{t} x_{t}\right) d t>0
$$

Analogamente, se $x \equiv-M$, então $\int_{0}^{T} N x(t) d t<0$. Assim, concluímos a prova de que a condição (ii) é satisfeita.

Para finalizar, vejamos que a condição (iii) é satisfeita, ou seja, $\operatorname{deg}\{J Q N, \Omega \cap \operatorname{Ker} L, 0\} \neq 0$, sendo $J$ um isomorfismo entre $\operatorname{Im} Q$ e $\operatorname{Ker} L$. Note que $\operatorname{Im} Q$ e $\operatorname{Ker} L$ podem ser identificados $($ com $\mathbb{R})$ e, portanto, podemos tomar $J$ como sendo o operador identidade entre $\operatorname{Im} Q$ e $\operatorname{Ker} L$. Assim, devemos mostrar que $\operatorname{deg}\{Q N, \Omega \cap \operatorname{Ker} L, 0\} \neq 0$. Para isso, usaremos a propriedade da invariância do grau topológico por homotopia.

Defina $H:(\bar{\Omega} \cap \operatorname{Ker} L) \times[0,1] \longrightarrow \mathbb{R}$, pondo

$$
H(x, \lambda)=(1-\lambda) x+\frac{\lambda}{T} \int_{0}^{T} N x(t) d t
$$

Note que $\Omega \cap \operatorname{Ker} L=(-M, M)$ e, consequentemente,

$$
\partial(\Omega \cap \mathbb{R}) \times[0,1]=\{-M, M\} \times[0,1] .
$$

Na sequência, veremos que $0 \notin H(\{-M, M\} \times[0,1])$. Fixado $\lambda \in[0,1]$ e usando as definições de $H$ e de $N$, obtemos

$$
H(M, \lambda)=(1-\lambda) M+\frac{\lambda}{T} \int_{0}^{T} \beta(t)^{-1} f\left(t, \beta_{t} M\right) d t
$$


Note que a primeira parcela do lado direito de (3.28) é não negativa. Vejamos que o mesmo vale para a segunda parcela. Como $M>d$, então pela hipótese $(H 2)$, temos

$$
f\left(t, \beta_{t} M\right)>0, \quad t \in[0, T]
$$

e, por conseguinte,

$$
\frac{\lambda}{T} \int_{0}^{T} \beta(t)^{-1} f\left(t, \beta_{t} M\right) d t \geq 0
$$

Note que vale a igualdade em (3.29) somente quando $\lambda=0$, caso em que a primeira parcela do lado direito de (3.28) é positiva. Por outro lado, a primeira parcela do lado direito de (3.28) somente se anula quando $\lambda=1$, caso em que a segunda parcela é positiva. Dessa forma, concluímos que $H(M, \lambda)>0$, como queríamos demonstrar. De modo análogo, mostra-se que $H(-M, \lambda)<0$ para cada $\lambda \in[0,1]$. Isto conclui a prova de que $0 \notin H(\partial(\Omega \cap \operatorname{Ker} L) \times[0,1])$ e, portanto, $\operatorname{deg}(H(\cdot, 0), \Omega \cap \operatorname{KerL}, 0)$ e $\operatorname{deg}(H(\cdot, 1), \Omega \cap \operatorname{KerL}, 0)$ estão bem definidos. Além disso, pela invariância do grau topológico por homotopia, temos

$$
\operatorname{deg}(H(\cdot, 0), \Omega \cap \operatorname{KerL}, 0)=\operatorname{deg}(H(\cdot, 1), \Omega \cap \operatorname{KerL}, 0) .
$$

Por outro lado, para cada $x \in \Omega \cap \operatorname{KerL}$, temos

$$
H(x, 0)=x \quad \text { e } \quad H(x, 1)=\frac{1}{T} \int_{0}^{T} N x(t) d t=Q N x(t)
$$

Portanto, de (3.30) e (3.31), obtemos

$$
\begin{aligned}
\operatorname{deg}(Q N, \Omega \cap \operatorname{Ker} L, 0) & =\operatorname{deg}(H(\cdot, 1), \Omega \cap \operatorname{KerL}, 0)=\operatorname{deg}(H(\cdot, 0), \Omega \cap \operatorname{Ker} L, 0) \\
& =\operatorname{deg}(\operatorname{Id}, \Omega \cap \operatorname{Ker} L, 0)=1,
\end{aligned}
$$

e a demonstração está completa. 
Em vista do Teorema 3.2 e dos Lemas 3.8 a 3.12 podemos provar o Teorema 3.5.

Demonstração. (do Teorema 3.5) Suponha que as condições (H1) a (H4) estejam satisfeitas. Pelos Lemas 3.8 a 3.12 e pelo Teorema 3.2 a equação $L x=N x$ possui pelo menos uma solução $z:[0, T] \longrightarrow \mathbb{R}$, pertencente a $W \cap \bar{\Omega}$. Considere a função $x:[-r,+\infty) \longrightarrow \mathbb{R}$ dada por $x(t)=z(t)$ se $t \in[0, T], x(t)=x(t+T)$ se $t \in[-r, 0]$ e $x(t)=x(t-n T)$ se $t \in(n T,(n+1) T]$. Então a função $x$ é $T$-periódica, é continuamente diferenciável nos intervalos $\left[0, t_{1}\right],\left(t_{k}, t_{k+1}\right], k \in \mathbb{N}$ e, pela Definição 3.3, é uma solução de (3.7).

Neste momento, estamos em condições de enunciar o principal resultado desta seção.

Teorema 3.13. Se as condições (H1) a (H4) forem satisfeitas, então o problema (3.1) terá pelo menos uma solução T-periódica e continuamente diferenciável nos intervalos $\left[0, t_{1}\right],\left(t_{k}, t_{k+1}\right]$, $k \in \mathbb{N}$.

Demonstração. O resultado segue imediatamente do Teorema 3.5 e da primeira parte do Lema 3.4 .

\subsection{Unicidade de solução periódica}

Nesta seção, investigaremos a unicidade de solução $T$-periódica para o problema (3.1). Para tanto utilizaremos a segunda parte do Lema 3.4 que nos assegura que a unicidade de solução para a equação (3.7) implica no mesmo para o Prolema 3.1. Mostraremos que se a função $f$ for injetiva na segunda variável, então as hipóteses $(H 1)$ a $(H 4)$ implicarão na unicidade de solução $T$-periódica para a equação (3.7).

Teorema 3.14. Se a função $f$ for injetiva na segunda variável e se as condições $(H 1)$ a (H4) forem satisfeitas, então a equação (3.7) possuirá uma única solução T-periódica e continuamente diferenciável nos intervalos $\left[0, t_{1}\right],\left(t_{k}, t_{k+1}\right], k \in \mathbb{N}$. 
Demonstração. Suponha que para cada $t \in[0, T]$ a função $f(t, \cdot)$ seja injetiva e que as condições $(H 1)$ a (H4) estejam satisfeitas. Suponha ainda que $\varphi$ e $\psi$ sejam soluções $T$-periódicas da equação (3.7) e que, além disso, tais funções sejam continuamente diferenciáveis nos intervalos $\left[0, t_{1}\right],\left(t_{k}, t_{k+1}\right], k \in \mathbb{N}$. Vamos mostrar que a função $\zeta=\varphi-\psi$ é identicamente nula. Como $\varphi$ e $\psi$ são $T$-periódicas, basta mostrar que $\zeta \equiv 0$ em $[0, T]$. Para tanto, primeiramente mostraremos que $\zeta$ se anula em algum ponto de $[0, T]$ e, na sequência, que $\zeta$ é uma função constante.

Como $\varphi$ e $\psi$ são soluções de (3.7) então, para quase todo $t \in[0, T]$, temos

$$
\zeta^{\prime}(t)+B \beta(t)^{-1} \beta(t-\delta) \zeta^{\prime}(t-\delta)=h\left(t, \varphi_{t}\right)-h\left(t, \psi_{t}\right)
$$

Integrando de 0 a $T$ ambos os membros dessa igualdade, obtemos

$$
\int_{0}^{T} h\left(t, \varphi_{t}\right)-h\left(t, \psi_{t}\right) d t=0
$$

ou seja,

$$
\int_{0}^{T} \beta(t)^{-1}\left[f\left(t, \beta_{t} \psi_{t}\right)-f\left(t, \beta_{t} \varphi_{t}\right)\right] d t=0
$$

Por outro lado, pela hipótese $(H 1)$, a função

$$
t \mapsto f\left(t, \beta_{t} \psi_{t}\right)-f\left(t, \beta_{t} \varphi_{t}\right), \quad t \in[0, T]
$$

é contínua. Isto e (3.33) implicam que existe $\tau \in[0, T]$, de modo que

$$
f\left(\tau, \beta_{\tau} \psi_{\tau}\right)-f\left(\tau, \beta_{\tau} \varphi_{\tau}\right)=0
$$

Disso e da injetividade da função $f(\tau, \cdot)$, obtemos $\psi(\tau)=\varphi(\tau)$ e, portanto, $\zeta(\tau)=0$. Assim, para finalizar a demonstração de que $\zeta \equiv 0$, basta mostrarmos que $\zeta$ é uma função constante. 
Como $\zeta(\tau)=0$, então, pelas desigualdades triangular e de Hölder, temos

$$
|\zeta(t)|=\left|\zeta(\tau)+\int_{\tau}^{t} \zeta^{\prime}(s) d s\right| \leq \int_{0}^{T}\left|\zeta^{\prime}(s)\right| d s \leq \sqrt{T}\left\|\zeta^{\prime}\right\|_{2}, \quad t \in[0, T]
$$

Consequentemente,

$$
\|\zeta\|_{\infty} \leq \sqrt{T}\left\|\zeta^{\prime}\right\|_{2}
$$

Por outro lado, pela desigualdade triangular, a igualdade obtida em (3.32), implica

$$
\left|\zeta^{\prime}(t)\right| \leq\left|h\left(t, \varphi_{t}\right)-h\left(t, \psi_{t}\right)\right|+B \beta(t)^{-1} \beta(t-\delta)\left|\zeta^{\prime}(t-\delta)\right|
$$

Multiplicando por $\left|\zeta^{\prime}(t)\right|$ ambos os membros da expressão acima, obtemos

$$
\left|\zeta^{\prime}(t)\right|^{2} \leq\left|h\left(t, \varphi_{t}\right)-h\left(t, \psi_{t}\right)\right|\left|\zeta^{\prime}(t)\right|+B \beta(t)^{-1} \beta(t-\delta)\left|\zeta^{\prime}(t-\delta)\right|\left|\zeta^{\prime}(t)\right|
$$

Integrando de 0 a $T$ ambos os membros dessa expressão e utilizando a definição da função $h$, dada em (3.8), obtemos

$\left\|\zeta^{\prime}\right\|_{2}^{2} \leq \int_{0}^{T} \beta(t)^{-1}\left|f\left(t, \beta_{t} \varphi_{t}\right)-f\left(t, \beta_{t} \psi_{t}\right)\left\|\zeta^{\prime}(t)\left|d t+B \int_{0}^{T} \beta(t)^{-1} \beta(t-\delta)\right| \zeta^{\prime}(t-\delta)\right\| \zeta^{\prime}(t)\right| d t$.

Disto, das hipóteses (H3) e (H4), da desigualdade de Hölder e da majoração obtida em (3.34), resulta

$$
\begin{aligned}
\left\|\zeta^{\prime}\right\|_{2}^{2} & \leq b \int_{0}^{T}\left|\varphi(t)-\psi(t)\left\|\zeta^{\prime}(t)\left|d t+B L l^{-1} \int_{0}^{T}\right| \zeta^{\prime}(t-\delta)\right\| \zeta^{\prime}(t)\right| d t \\
& \leq b \sqrt{T}\|\zeta\|_{\infty}\left\|\zeta^{\prime}\right\|_{2}+B L l^{-1}\left\|\zeta^{\prime}\right\|_{2}^{2} \\
& \leq b T\left\|\zeta^{\prime}\right\|_{2}^{2}+B L l^{-1}\left\|\zeta^{\prime}\right\|_{2}^{2} .
\end{aligned}
$$


Portanto, como pela hipótese $0<b<\left(1-B L l^{-1}\right) / T$, então $\zeta^{\prime} \equiv 0$ e, por conseguinte, $\zeta$ é constante, como queríamos mostrar.

Neste momento, podemos enunciar o resultado principal deste capítulo.

Teorema 3.15. Se f for injetiva na segunda variável e se as condições (H1) a (H4) forem satisfeitas, então o Problema (3.1) possuirá no máximo uma solução T-periódica.

Demonstração. Consequência imediata do Teorema 3.14 e da segunda parte do Lema 3.4. 


\section{Referências Bibliográficas}

[1] A. Antonevič, A. V. Lebedev, M. Belousov, Functionals Differential Equations, Taylor and Francis, 1998.

[2] N. V. Azbelev, L. Rakhmatullina, Introduction to the Theory of Functionals Differential Equations: Methods and Applications, Hindawi Publishing Corporation, 2007.

[3] Chuanzhi Bai, Global exponential stability and existence os periodic solution of CohenGrossberg type neural networks with delays and impulses, Nonlinear Analisys: Real World Applications, 9 (2008), 747-761.

[4] D. Baĭnov, P. S. Simeonov, Impulsive Diferential Equations: Periodic Solutions and Applications, Longman Scientific, 1993.

[5] D. Baïnov, V. Covachev, Impulsive Differential Equations with a Small Parameter, World Scientific, 1994.

[6] D. Bă̈nov, S. I. Kostadinov, Abstract Impulsive Differential Equations, Descartes Press, 1996.

[7] M. Benchohra, S. Ntouyas, Impulsive Differential Equations and Inclusions, Hindawi Publishing Corporation, 2006.

[8] H. Berestycki, Méthodes Topologiques et Problèmes Aux Limites non Linéaires, Thèse de Doctorat, l’Université de Paris VI, 1975.

[9] O. B. de Almeida, Teoria do Grau e Aplicações, Dissertação de Mestrado do Programa de Pós-Graduação em Matemática - CCT - UFCG, (2006). 
[10] K. Deimling, Nonlinear Functional Analysis, Springer-Verlag, 1985.

[11] Xuanlong Fan, Yongkun Li, Positive periodic solutions of neutral functional differential equations with a parameter and impulse, Electronic Journal of Differential Equations, 38 (2008), 1-8.

[12] I. Fonseca; W. Gangbo, Degree Theory in Analysis and Applications, Oxfords Science Publications, Clarendon Press, Oxford, 1995.

[13] D. Franňková, Regulated functions, Mathematica Bohemica, 1 (1991), 20-59.

[14] R. E. Gaines ; J. Mawhin, Coincidence Degree and Nonlinear Differential Equations, Lecture Notes in Math. 568, Springer-Verlag, 1977.

[15] Zhanji Gui, Xiao-Song Yang, Weigao Ge, Existence and global exponential stability of periodic solutions of recurrent cellular neural networks with impulses and delays, Mathematics and Computers in Simulation, 79 (2008), 14-29.

[16] J. K. Hale, S. M. Verduyn Lunel, Introduction to Functional Differential Equations. Applied Mathematical Sciences, 99. Springer-Verlag, 1993.

[17] C. S. Hönig, Volterra Stieltjes-Integral Equations. Functional Analytic Methods; Linear Constraints. Mathematics Studies, No. 16, North-Holland Publishing Co., AmsterdamOxford; American Elsevier Publishing Co., New York, 1975.

[18] Hai-Feng Huo, Wan-Tong Li, Positive periodic solution of a neutral delay competition model with impulses, Applicable Analysis, 84 (11) (2005), 1103-1116.

[19] Hai-Feng Huo, Existence of positive periodic solutions of a neutral delay Lotka-Volterra System with impulses, Computers and Mathematics with Applications, 48 (2004), 18331846. 
[20] Junguo Jia, Miansen Wang, Meili Li Periodic solutions for impulsive delay differential equations in the control model of plankton allelopathy, Chaos Solutions \& Fractals, 32 (2) (2007), 962-968.

[21] A. V. Kim, Functional Differential Equations, Kluwer, 1996.

[22] V. Kolmanovskii, A. Myshkis Introduction to the Theory and Applications of Functional Differential Equations, Kluwer Academic Publishers, Dordrecht, 1999.

[23] Yang Kuang, Delay Differential Equations With Applications in Population Dynamics, Academic Press, INC., London, 1993.

[24] V. Lakshmikantham, D. D. Bainov, P.S. Simeonov, Theory of Impulsive Differential Equations, World Scientific Publishing Co. Pte. Ltd, Singapure, 1989.

[25] Jianli Li, Jianhua Shen, Periodic boundary value problems for delay differential equations with impulses Journal of Computational and Applied Mathematics, 193 (2006), 563-573.

[26] Jing-Wen Li, Sui Sun Cheng Periodic solutions os a second order forced sublinear differential equation with delay, Applied Mathematics Letters, 18 (2005), 1373-1380.

[27] Meili Li, Chunhai Kou, Yougrui Duan, The existence of periodic solution of impulsive functional differential equation with infinite delay, J. Appl. Math. Comput., 29 (2009), $341-348$.

[28] Wan-Tong Li, Hai-Feng Huo, Global attractivity of positive periodic solutions for an impulsive delay periodic model of respiratory dynamics, Journal of Computational and Applied Mathematics, 174 (2005), 227-238.

[29] Xinzhi Liu, George Ballinger, Boundness for impulsive delay differential equations and applications to population growth models, Nonlinear Analisys, 53 (2003), 1041-1062. 
[30] Bing-wen Liu, Li-hong Huang, Existence and uniqueness of periodic solutions for a kind os second order neutral funcional differential equations with constante delays, Acta Mathematicae Applicatae Sinica, 22 (2)(2006), 287-296.

[31] J. Mallet-Paret, R. D. Nussbaum, P. Paraskevopoulos, Periodic solutions for functional differential equations with multiple state-dependent time lags, Journal of the Juliusz Schauder Center 3 (1994), 101-162.

[32] F. Ren, J. Cao, Periodic solutions for a class of higher-order Cohen-Grossberg type neural networks with delays, Computers and Mathematics with Applications, 54 (2007), 826-839.

[33] A. M. Samoǐlenko, N. A. Perestíuk, Impulsive Differential Equations World Scientific, 1996.

[34] I. Stamova, Stability Analysis of Impulsive Functional Differential Equations, Walter de Gruyter GmbH \& Co, 2009.

[35] Hui Wang, Xiaofeng Liao, Chuandong Li, Existence and exponential stability of periodic solution of BAM neural networks with impulse and time-varyng delay, Chaos, Solitons \& Fractals 33 (2007), 1028-1039.

[36] Jurang Yan, Aimin Zhao, J. J. Nieto, Existence and global attractivity of positive periodic solution of periodic single-species impulsive Lotka-Volterra Systems, Mathematical and Computer Modelling, 40 (2004), 509-518. 